\title{
Vermittlerstrategien und Arbeitsmarkterfolg - Evidenz aus kombinierten Prozess- und Befragungsdaten
}

\author{
Bernhard Boockmann · Christopher Osiander • \\ Michael Stops
}

Online publiziert: 11. Juni 2014

(C) Institut für Arbeitsmarkt- und Berufsforschung 2014

Zusammenfassung Trotz eines umfangreichen Regelwerks verbleiben den Agenturen für Arbeit und ihren Vermittlerinnen und Vermittlern erhebliche Gestaltungsspielräume bei der Umsetzung der Arbeitsmarktpolitik. In dieser Studie untersuchen wir anhand von kombinierten Befragungs- und Prozessdaten, welche Strategien und Ansätze am wirkungsvollsten für die Integration von Arbeitslosen im Rechtskreis der Arbeitslosenversicherung (SGB III) sind. Die dazu erforderliche Datenverknüpfung wird erstmals für Deutschland vorgenommen. Die meisten Effekte sind in der Größenordnung recht gering und nur für Teilzeiträume statistisch signifikant. Es stellt sich heraus, dass Vermittler, die die schnelle Vermittlung als wichtigstes Ziel sehen, tendenziell bessere Integrationsergebnisse erzielen als Vermittler, die andere Zielsetzungen für wichtiger halten. Die Bereitschaft zur Nutzung von Sperrzeiten wirkt sich schwach signifikant positiv auf den Abgang aus Arbeitslosigkeit aus, nicht jedoch auf die Wahrscheinlichkeit, eine Beschäftigung aufzunehmen. Häufigere Kontakte zwischen Vermittlern und Arbeitslosen haben tendenziell

Prof. Dr. B. Boockmann $(\bowtie)$

Institut für Angewandte Wirtschaftsforschung (IAW),

University of Tübingen,

Ob dem Himmelreich 1,

72074 Tübingen, Deutschland

E-Mail: bernhard.boockmann@iaw.edu

C. Osiander $\cdot$ M. Stops

Institut für Arbeitsmarkt- und Berufsforschung (IAB),

Regensburger Straße 104,

90478 Nürnberg, Deutschland

E-Mail: christopher.osiander@iab.de

M. Stops

E-Mail: michael.stops@iab.de eine positive Wirkung auf den Abgang aus der Arbeitslosigkeit. Wird internen Weisungen bei der Vergabe von Maßnahmen innerhalb der Agenturen ein höherer Stellenwert gegenüber anderen Kriterien eingeräumt, verbessern sich die Integrationschancen der Betreuten zu Beginn der Arbeitslosigkeit.

Schlüsselwörter Arbeitsvermittler - Arbeitslosigkeit · Integration · Bundesagentur für Arbeit · SGB III · Treatment-Effekte

JEL-Code J08 $\cdot$ J64 $\cdot$ J68

\section{Caseworkers' strategies and clients' labor market outcomes}

Abstract In spite of extensive regulations, Germany's job offices and their caseworkers have substantial discretion in implementing labor market policies. Based on the analysis of combined survey and administrative data, this study compares the effectiveness of different strategies aimed at re-integrating job seekers in the unemployment insurance system (Social Code III) into regular employment. The data merger is an innovative contribution of our paper, since it is done for the first time for German data. Most of the estimated treatment effects are moderate in size and statistically significant only for parts of the unemployment duration. We find that caseworkers who emphasize rapid integration into work as their primary goal tend to achieve better results for their clients than caseworkers who consider other goals important. While the willingness to impose sanctions weakly significantly increases the probability to leave unemployment, it does not have a positive impact on the probability to find employment. Furthermore, 
frequent contacts between caseworker and job seeker tend to have positive effects in terms of exit from unemployment. Finally, caseworkers who consider internal regulations important when they allocate job seekers to programs of ALMP tend to achieve better integration outcomes at the beginning of clients' unemployment spells.

Keywords Placement service - Unemployment · Integration - Federal Employment Agency · Unemployment Insurance $\cdot$ Treatment effects

\section{Einleitung}

Die öffentliche Arbeitsverwaltung wurde während des letzten Jahrzehnts in vielen Industrieländern neu gestaltet. ${ }^{1}$ Dadurch rückte auch die Rolle der Mitarbeiter $^{2}$ in der Arbeitsvermittlung zunehmend in den Mittelpunkt der Diskussionen über erfolgreiche Arbeitsmarktpolitik. Trotz dichter Regelwerke zur Organisation der öffentlichen Arbeitsvermittlung verbleiben den Agenturen für Arbeit und ihren Beschäftigten vor Ort Handlungsspielräume bei der Beratung und Betreuung der Arbeitslosen. Sie bestimmen mit, wer Zugang zu welchen Maßnahmen der aktiven Arbeitsmarktpolitik erhält; zugleich sind sie für die Aktivierung zuständig und müssen normwidriges Verhalten erkennen. Die Einstellungen und Strategien von Arbeitsvermittlern könnten dadurch nicht weniger wichtig sein als die Ausgestaltung der arbeitsmarktpolitischen Instrumente durch Gesetze und Handlungsanweisungen.

In diesem Beitrag untersuchen wir, wie die Vermittler in den Agenturen für Arbeit in Deutschland durch ihre Einstellungen und Strategien die Chancen ihrer Kunden beeinflussen, ihre Arbeitslosigkeit $\mathrm{zu}$ beenden und ein Beschäftigungsverhältnis aufzunehmen. Dies ist aus wissenschaftlicher Sicht aus zwei Gründen bedeutsam: erstens existiert (noch) keine in sich geschlossene Handlungs- oder Prozesstheorie der Arbeitsvermittlung und zweitens sind empirische Erkenntnisse zum Einfluss von Vermittlern auf die Arbeitsmarktergebnisse der von ihnen betreuten Arbeitslosen rar. Genauere Kenntnisse hierüber sind relevant für die Politik und Arbeitsverwaltung. Dies trifft auch deshalb zu, weil bei gegebenem finanziellem Aufwand unterschiedliche Herangehensweisen keine direkten zusätzlichen Mehrkosten für die Erbringung der Vermittlungsdienst-

\footnotetext{
${ }^{1}$ Das beinhaltet substanzielle Reformen der Organisation (de Koning 2004; Eichhorst et al. 2008; Knuth et al. 2004; für Deutschland Vaut 2004; Sell 2006; Schütz 2008), von Anreizstrukturen (Knuth et al. 2004) und Managementpraktiken (Weishaupt 2010; für Deutschland Sell 2006).

${ }^{2}$ Im Folgenden werden möglichst geschlechtsneutrale Formulierungen gewählt. Eine etwaige Beschränkung auf die männliche Form erfolgt ausschließlich aus Gründen der Lesbarkeit.
}

leistungen der Arbeitsverwaltung erzeugen, im Gegensatz zu Entscheidungen für den Einsatz von arbeitsmarktpolitischen Maßnahmen.

Bisherige empirische Wirkungsanalysen beschäftigen sich mit der Frage, wie wichtig Intensität und Qualität der Beratung für den Integrationserfolg von Arbeitslosen sind. Darüber hinaus liegen einzelne Studien vor, in denen Elemente des Vermittlungsprozesses oder Handlungsweisen der Vermittler untersucht wurden, beispielsweise der Stellenwert schneller Vermittlung gegenüber der Zuweisung in Maßnahmen, die Nutzung von Sanktionen ${ }^{3}$ und die Berücksichtigung von Wünschen der Arbeitslosen. $\mathrm{Zu}$ anderen Unterschieden zwischen Vermittlern, beispielsweise im Hinblick auf die Selbstwahrnehmung der eigenen Tätigkeit oder die Bedeutung der Handlungsautonomie, liegen allenfalls theoretische Hypothesen oder qualitative Ergebnisse vor, jedoch keine quantitativen Wirkungsstudien.

Für unsere Untersuchung verwenden wir einen neuartigen Datensatz, der eine standardisierte Befragung von Vermittlern mit den administrativen Daten der Bundesagentur für Arbeit (BA) über ihre Kunden verknüpft. Die Möglichkeit einer solchen Verknüpfung ist ein wesentliches Ergebnis unserer Studie, das auch künftig in der Arbeitsmarktforschung genutzt werden kann. Durch den kombinierten Datensatz verfügen wir über ein breites Spektrum an Informationen über Strategien und Einstellungen der Arbeitsvermittler. Auf dieser Grundlage führen wir eine Wirkungsanalyse durch, die den Einfluss des Vermittlerhandelns ${ }^{4}$ auf den Wiedereingliederungserfolg der von den Vermittlern betreuten Kunden untersucht. Wir verwenden ein statistisches Matching-Verfahren, mit dem eine Kontrollgruppe aus den verfügbaren Daten generiert wird. Damit kann analysiert werden, ob sich die Arbeitsmarktergebnisse von Kunden unterscheiden, die von Vermittlern mit unterschiedlichen Handlungsstrategien betreut werden, aber ansonsten sehr ähnliche Merkmale aufweisen. Unter bestimmten plausiblen Annahmen lassen sich die Ergebnisse als kausaler Einfluss der Strategien und Handlungsweisen auf den Arbeitsmarkterfolg interpretieren.

Die ökonometrischen Schätzungen ergeben, dass die Beendigung von Arbeitslosigkeit und die Aufnahme einer Beschäftigung durch die Kunden von einigen dieser Ein-

\footnotetext{
${ }^{3}$ Mit Sanktionen bezeichnen wir hier alle gesetzlich vorgesehenen Maßnahmen zur Kürzung im Leistungsbezug bei Arbeitslosigkeit im Falle regelwidrigen Verhaltens. In Deutschland sind das die in der Arbeitslosenversicherung vorgesehenen Sperrzeiten sowie Sanktionen, die im Bereich der Grundsicherung geregelt sind. In unserer eigenen empirischen Analyse beziehen wir uns immer auf Sperrzeiten nach dem SGB III.

${ }^{4}$ Hier und im Folgenden verstehen wir unter „Vermittlerhandeln“ die von den befragten Fachkräften beschriebenen Handlungsweisen bzw. die sich aus den beschriebenen Strategien und Einstellungen ergebenden Handlungsweisen bei der Erfüllung der Aufgaben in der Arbeitsvermittlung.
} 
stellungen und Handlungsweisen der Vermittler signifikant beeinflusst werden.

Der Beitrag ist wie folgt aufgebaut. In Abschnitt 2 werden die in der Evaluationsliteratur bisher vorhandenen Ergebnisse zu den Wirkungen des Vermittlerhandelns zusammengefasst. Abschnitt 3 beschreibt die Daten. In Abschnitt 4 wird das Vorgehen für die Wirkungsanalyse erläutert. Abschnitt 5 stellt die empirischen Befunde vor. Abschnitt 6 fasst die Resultate zusammen und gibt einen Ausblick auf weitere Forschung.

\section{Erkenntnisse aus früheren Studien}

\subsection{Intensität und Qualität der Betreuung}

Intensität und Qualität der Betreuung können unterschiedlich operationalisiert werden, beispielsweise über die Kontaktdichte zwischen Vermittler und Arbeitsuchenden, aber auch über die quantitative Betreuungsrelation (Arbeitsuchende pro Vermittler). Pedersen et al. (2012) berichten Ergebnisse eines sozialen Experiments in Dänemark, bei dem eine hohe Kontaktdichte von Vermittlern und Arbeitslosen die Integrationschancen beträchtlich steigert. Van den Berg et al. (2012) untersuchen ebenfalls die Wirkung häufiger Interaktionen zwischen Vermittlern und Kunden mit administrativen Daten aus Dänemark. Sie ermittelten für die Woche, in denen ein Beratungsgespräch stattfand, sowie für die Folgezeit höhere Übergangsraten in Beschäftigung, wobei die Ergebnisse zwischen Altersgruppen und Geschlecht differenziert ausfallen. Für Deutschland weisen Hainmueller et al. (2011) statistisch signifikante Effekte der quantitativen Betreuungsrelation nach. Weniger Fälle pro Betreuer führen zu einer Verringerung der regionalen Arbeitslosenquote, einer Verkürzung der Arbeitslosigkeitsdauer und einer höheren Beschäftigungsquote.

Die Ausgestaltung der Betreuung ist ebenfalls von Bedeutung. Experimente in verschiedenen Ländern (Kanada, Schweden, Vereinigtes Königreich und die USA) weisen darauf hin, dass über das übliche Maß hinaus intensivierte Vermittlungsbemühungen (zusätzliche Bewerbungscoachings, höhere Kontaktdichte) verbunden mit verstärktem Monitoring und Sanktionen zu einer schnelleren Wiedereingliederung in Beschäftigung führen (Martin und Grubb 2001).

\subsection{Vermittlungsorientierung}

Für Schweden schätzt Lagerström (2011) die Wirkungen jedes einzelnen Vermittlers in der Stichprobe auf die zukünftigen Beschäftigungschancen, Löhne und Einkommen der betreuten Arbeitslosen. Er zeigt, dass sich die Wahrscheinlichkeit, etwa ein Jahr später erwerbstätig zu sein, je nach zugewiesenem Vermittler erheblich unterscheiden kann.
Erfolgreiche Vermittler konzentrieren sich Lagerström (2011) zufolge eher auf die Stellensuche, anstatt den Arbeitsuchenden Maßnahmen der aktiven Arbeitsmarktpolitik zuzuweisen. Dieses Resultat entspricht den Ergebnissen anderer Studien, wonach insbesondere Leistungen, die auf die Arbeitssuche und Vermittlung gerichtet sind, positiv auf die Beschäftigungschancen wirkten (siehe insbesondere die Meta-Studien von Kluve 2010; Card et al. 2010 sowie den Überblick von Brown und Koettl 2012), obgleich eine kausale Interpretation des Ergebnisses starken Annahmen unterliegt.

Im Zusammenhang mit verstärkten Vermittlungsbemühungen im Rahmen der Betreuung von Arbeitslosen stellt sich auch die Frage nach der Nachhaltigkeit der Wirkungen auf die Aufnahme von Beschäftigung und die Beendigung der Arbeitslosigkeit. Wenn die Vermittlung in eine Beschäftigung den alleinigen Erfolgsmaßstab darstellt, werden möglicherweise andere wünschenswerte Ziele - beispielsweise die Qualität der geschaffenen Beschäftigungsverhältnisse - vernachlässigt. So könnten zusätzlich erreichte Integrationen nicht nachhaltig sein. ${ }^{5}$ Des Weiteren wäre kaum auszuschließen, dass sich Berater, deren Erfolgsmaßstab allein die Zahl der Vermittlungen ist, stark auf die Kunden mit den besten Aussichten am Arbeitsmarkt konzentrieren und längerfristige Bemühungen um die Qualifizierung und Beseitigung von Vermittlungshemmnissen bei arbeitsmarktferneren Personen vernachlässigen. Dieses so genannte „cream skimming“ ist jedoch nicht effektiv, da Wunsch (2013) zufolge Hilfen bei der Arbeitssuche und Vermittlung gerade für arbeitsmarktfernere Kunden mit geringeren Chancen auf eine Beschäftigung wirkungsvoll sind.

Ferner sind unterschiedliche Zeitpunkte denkbar, zu denen (verstärkte) Vermittlungsbemühungen angesetzt werden, beispielsweise schon vor dem drohenden Eintritt in Arbeitslosigkeit, kurz nach deren Beginn oder bei einem Wechsel des Vermittlers bei längerer Arbeitslosigkeitsdauer. Der Zeitpunkt der Intervention kann deren Wirksamkeit beeinflussen: Nach den Befunden von Kastoryano und van der Klaauw (2011) sowie Wunsch (2013) sind diese vermehrten Anstrengungen zu Beginn der jeweiligen individuellen Arbeitslosigkeit am effektivsten.

\subsection{Monitoring der Arbeitsuchenden und Sanktionen}

Sowohl die mikroökonomisch fundierte Prinzipal-AgentenTheorie als auch die Suchtheorie legen den Schluss nahe, dass häufiges und effektives Monitoring bei der Stellensu-

\footnotetext{
${ }^{5}$ Ferner wird angesprochen, inwieweit die neu geschaffenen Beschäftigungsverhältnisse zusätzlich sind oder bestehende Beschäftigungsverhältnisse verdrängen (siehe beispielsweise Gautier et al. 2012). Da dieser Zusammenhang in der folgenden Wirkungsanalyse nicht berücksichtigt wird, gehen wir darauf nicht weiter ein.
} 
che geeignet ist, die Wiederaufnahme einer Beschäftigung $\mathrm{zu}$ beschleunigen und damit die Arbeitslosigkeitsdauer zu verkürzen (Behncke et al. 2010a; van den Berg und van der Klaauw 2005; van den Berg et al. 2012). Generell verfügen Vermittler mit Sanktionen und Sanktionsdrohungen über Instrumente, mit denen sie die Opportunitätskosten längerer Arbeitslosigkeit für die Kunden erhöhen können. Sind die Opportunitätskosten niedrig, haben Arbeitslose einen Anreiz, wenig passende Stellenangebote abzulehnen, um auf bessere zu warten. Durch intensivere Stellensuche und die Androhung von Sanktionen - so die Annahme - steigen die Opportunitätskosten des Wartens und der Anreiz, vorliegende Stellenangebote unmittelbar anzunehmen.

Suchtheoretische Argumente zielen auf die Anreizproblematik von Versicherungsleistungen ab, die opportunistisches Verhalten begünstigen, damit die Leistung in Anspruch genommen werden kann (,moral hazard“, vgl. bspw. Müller und Oschmiansky 2005). Sanktionen oder ihre Androhung sollen zu intensiveren Bewerbungsaktivitäten oder zu sinkenden Anspruchslöhnen führen. ${ }^{6}$

Müller und Oschmiansky (2005) zeigen, dass in Deutschland regionale Unterschiede bei der Implementation von Sanktionen existieren. So wird bei schlechter Arbeitsmarktlage seltener sanktioniert. Die Verschärfung von Sanktionsregelungen im Zuge der Hartz-Reformen hat zudem für einen Niveauanstieg bei Sanktionsquoten geführt, die außerdem regional deutlich heterogener ausfallen als vor den Reformen. Boockmann et al. (2009) nutzen diese regionale Variation als Instrumentenvariable für die tatsächliche Verhängung von Sanktionen. Für den Bereich des SGB II zeigen sie, dass sich die Übergangswahrscheinlichkeit in bedarfsdeckende Beschäftigung nach der Sanktion deutlich erhöht.

\subsection{Kooperation mit den Arbeitslosen und Qualität der Betreuungsbeziehung}

Vermittler können die Bedürfnisse und Wünsche der Kunden im Beratungsprozess berücksichtigen. Dies kann zu einer Atmosphäre von Respekt und gegenseitigem Vertrauen führen, die ein reziprokes Verhalten der Kunden befördert. Für die Schweiz zeigen Behncke et al. (2010a) jedoch, dass Vermittler, die nicht auf die Wünsche der Arbeitslosen eingehen, sondern ihre Vermittlungsstrategie und die Zuweisung $\mathrm{zu}$ arbeitsmarktpolitischen Maßnahmen unabhängig von den Arbeitslosen festlegen, die durchschnittliche Integrationswahrscheinlichkeit ihrer Kunden über den Erfolg anderer Vermittler hinaus signifikant erhöhen.

\footnotetext{
${ }^{6}$ Empirische Evidenz zur Wirkung von Sanktionen enthalten unter anderem Arni et al. (2013); Boockmann et al. (2009); Hofmann (2012); Svarer (2011) und van den Berg et al. (2004).
}

In Behncke et al. (2010b) überprüfen dieselben Autoren, ob ein ähnlicher sozialer Hintergrund bei Arbeitsuchenden und Vermittlern - operationalisiert durch Geschlecht, Alter, Nationalität, Muttersprache, formales Bildungsniveau und andere Merkmale - zu größerem Vertrauen, mehr wahrgenommener Fairness und damit zu einer höheren Integrationswahrscheinlichkeit führt. Dem liegt die Hypothese zugrunde, dass Akteure mit einem ähnlichen sozialen Hintergrund mit ähnlichen verbalen und nonverbalen Konzepten kommunizieren, was zu Kooperation auf der Basis eines „gift exchange“ und zu reziprokem Handeln führt. Die Autoren finden einen positiven Beschäftigungseffekt in der Größenordnung von etwa vier Prozentpunkten, wenn Vermittler und Arbeitsuchender zur selben sozialen Gruppe gehören.

\subsection{Handlungsspielräume und Normakzeptanz}

Der Grad der von der Organisation eingeräumten Handlungsspielräume ist ein weiterer Aspekt, der die tägliche Arbeit von Vermittlern kennzeichnet (Lipsky 1980). Lagerström (2011) konstatiert, dass der Einfluss individueller Charakteristika des Vermittlers umso größer sein dürfte, je größer die Handlungsspielräume sind, über die er verfügt oder die er tatsächlich nutzt. Die Spielräume werden durch einheitliche Normen, aber auch durch formale Regelungen eingeschränkt, die sich von Organisation zu Organisation unterscheiden können.

So existieren beispielsweise interne Weisungen, die festlegen, welche Kundengruppen in bestimmte Maßnahmen der aktiven Arbeitsmarktpolitik zugewiesen werden können. Dies kann die Möglichkeiten für Vermittler einschränken, ihre eigenen Erfahrungen bei der Beratung von Kunden einfließen zu lassen, was unter Umständen nicht effektiv für das Integrationsziel ist. Andererseits ist denkbar, dass diese Regeln Orientierung vermitteln und dadurch das Vermittlerhandeln effektiver machen. So argumentieren Weatherall und Markwardt (2010), dass eine formalere Herangehensweise die Beschäftigungsfähigkeit der Kunden erhöht. Insgesamt ist theoretisch nicht eindeutig geklärt, welche Wirkung Handlungsspielräume haben.

\subsection{Zwischenfazit}

Die existierenden Studien benennen Rahmenbedingungen der öffentlichen Arbeitsvermittlung, die ausschlaggebend für den Vermittlungserfolg sein könnten. Erstens sind eine hohe Kontaktdichte zwischen Vermittler und Arbeitslosem sowie eine niedrige Betreuungsrelation von hoher Bedeutung für den (positiven) Vermittlungserfolg. Zweitens zeigt sich, dass Leistungen, die auf die Arbeitssuche und Vermittlung gerichtet sind und solche, die zu Beginn der Arbeitslosigkeit ansetzen, hilfreich sind. Sanktionen können die 
Beendigung von Arbeitslosigkeit bzw. den Übergang in Beschäftigung positiv beeinflussen, wobei mögliche negative Nebeneffekte noch weitgehend unerforscht sind. Strategien von Arbeitsvermittlern und ein ähnlicher sozialer Hintergrund bei Vermittlern und Arbeitslosen könnten ebenfalls Stellschrauben zur Verbesserung der Vermittlungsbemühungen sein. Offen blieb bislang, welchen Einfluss die unterschiedliche Nutzung verfügbarer Handlungsspielräume oder unterschiedliche Präferenzen hierüber haben.

\section{Befragung der Vermittler}

Die Daten für diese Studie stammen aus einer Befragung von Vermittlern in Agenturen für Arbeit ${ }^{7}$, die mit administrativen Daten der BA über Arbeitslose kombiniert werden. Diese Verknüpfung wird für Deutschland zum ersten Mal vorgenommen. In Abschnitt 3.1 beschreiben wir die Inhalte der Befragung, in Abschnitt 3.2 die administrativen Daten und die Verknüpfung der beiden Datenquellen. Abschnitt 3.3 enthält einige deskriptive Statistiken des kombinierten Datensatzes.

\subsection{Inhalte und Durchführung der Vermittlerbefragung}

Aufbauend auf den Befunden der im letzten Abschnitt vorgestellten Studien wird im Fragebogen eine Vielzahl von Themen aufgegriffen. Im Zentrum stehen die Anwendung bestimmter Strategien im Vermittlungsprozess und Einschätzungen $\mathrm{zu}$ vermittlungsrelevanten Themen. Daneben werden der berufliche Hintergrund der Befragten, das Arbeitsumfeld sowie persönliche Merkmale (Berufserfahrung, eigene Erfahrungen mit Arbeitslosigkeit, Netzwerke, soziodemografische Merkmale) erhoben.

Die Fragen zu Strategien und Einschätzungen umfassen vor allem die Themenbereiche Betreuungsintensität, Vermittlungsorientierung, Sanktionen, Kooperation mit den Arbeitslosen, den Grad der Standardisierung des Vorgehens, die Rolle von internen Weisungen und Standards. ${ }^{8}$

Bei der Formulierung der Fragen orientieren wir uns teilweise an der bereits zitierten Studie für die Schweiz (Frö-

\footnotetext{
${ }^{7}$ Befragungen wurden auch in Jobcentern durchgeführt, jedoch beschränkt sich die folgende Darstellung auf den Rechtskreis SGB III.

${ }^{8}$ Ein Fragebogenthesaurus ist in dem abschließenden Forschungsbericht zum Projekt zu finden (Boockmann et al. 2013), der unter http:// doku.iab.de/forschungsbericht/2013/fb0713.pdf zugänglich ist. Dort finden sich zudem detailliertere Angaben zu den Befragungsinhalten, als dies aus Platzgründen in diesem Beitrag möglich ist. Deskriptive Auswertungen finden sich außerdem in Boockmann et al. (2010). Um die Strategien und Handlungsspielräume möglichst vollständig $\mathrm{zu}$ erfassen, wurden Vermittlungsfachkräfte und Fallmanager sowie deren Teamleiter in die Befragung einbezogen. Die hier vorgestellten Ergebnisse stützen sich jedoch ausschließlich auf die Befragung der Vermittler aus der bewerberorientierten Vermittlung.
}

lich et al. 2007; Behncke et al. 2010a, b). Dabei nimmt der Fragebogen engen Bezug auf die Ausgestaltung der Arbeitsmarktpolitik in Deutschland und damit zur beruflichen Wirklichkeit der befragten Vermittler. Beispielsweise fragen wir nach der Vorgehensweise bei Instrumenten wie Kundendifferenzierung, Eingliederungsvereinbarungen, Profiling oder den arbeitsmarktpolitischen Maßnahmen des SGB III.

Dabei werden sowohl grundsätzliche Einstellungen, tatsächliche Handlungsweisen als auch Bewertungen dieser Handlungsweisen thematisiert. So gibt es Fragen zur Bedeutung von internen Weisungen für die Vergabe von arbeitsmarktpolitischen Maßnahmen und zum Grad, in dem diese Weisungen als nützlich oder hinderlich empfunden werden. Außerdem werden Einschätzungen über die Situation der Kunden erhoben. Anhand dieser Aussagen können beispielsweise eine kooperative und eine Druck ausübende Vorgehensweise unterschieden werden. Darüber hinaus werden Fragen zu den Zielen der Vermittlungstätigkeit, zur individuellen oder gesellschaftlichen Verursachung von Arbeitslosigkeit, zur Einstellung zu Sanktionen sowie zur Notwendigkeit von professioneller Distanz, von Einfühlungsvermögen und Empathie einbezogen.

Die Befragung wurde von März bis Juni 2009 in zehn Agenturen für Arbeit bzw. deren Geschäftsstellen durchgeführt. Bei der Auswahl der regionalen Einheiten wurden möglichst viele unterschiedliche regionale Arbeitsmarktlagen, abgebildet durch die Regionaltypen des IAB (Blien und Hirschenauer 2005), einbezogen. ${ }^{9}$ Das Organisationskonzept der BA sieht Vermittler vor, die Arbeitslose betreuen und solche, die bei Arbeitgebern Stellen akquirieren (sogenannter Arbeitgeberservice). Die Grundgesamtheit umfasst alle bewerberorientierten Vermittler, während die arbeitgeberorientierten Berater nicht einbezogen wurden.

Die Befragung wurde durch das IAB als Kombination aus schriftlicher und Online-Befragung durchgeführt, um eine möglichst große Beteiligung zu erreichen. ${ }^{10} \mathrm{Im}$ Bereich der Arbeitslosenversicherung (dem Rechtskreis SGB III) wurde der Fragebogen an 536 Vermittler versandt. Von diesen beteiligten sich 250 Personen an der Befragung. Die Online-Version des Fragebogens für Vermittler in den Agenturen war ab 26. März 2009 zur Beantwortung freigeschaltet, Ende der Feldzeit war der 15. Juni 2009. Alle potenziellen Teilnehmer erhielten im Lauf der Feldphase zwei Erinnerungsemails, in denen um Beteiligung an der Studie ersucht wurde, unabhängig davon, ob sie zum Zeitpunkt der Erinnerung bereits an der Umfrage teilgenommen hatten oder nicht. Insgesamt bearbeiteten 185 Personen den

\footnotetext{
${ }^{9}$ Siehe auch Tabelle 5 im Anhang.

${ }^{10}$ Die Vermittler konnten selbst entscheiden, den Fragebogen online ausfüllen oder die schriftliche Version zurückzusenden. Ausführliche methodische Erläuterungen finden sich im zum Projekt gehörenden Forschungsbericht (Boockmann et al. 2013).
} 
Fragebogen bis zur letzten Frage, was einer Beendigungsquote von knapp $35 \%$ entspricht. Für die Beteiligung an der Befragung ließ sich keine Selektivitätsanalyse auf Individualdatenbasis durchführen, da keine Individualdaten über nicht teilnehmende Vermittler vorliegen. Ersatzweise wurde hinsichtlich einiger Merkmale wie Alter, Qualifikation, Art des Arbeitsvertrags und Geschlecht ein Abgleich zwischen Angaben der Dienststatistik, die auf der Ebene der Arbeitsagenturen aggregiert wurden, und den in die Befragung einbezogenen Vermittlern durchgeführt. ${ }^{11}$

\subsection{Prozessdaten der BA und Verknüpfung mit den Befragungsdaten}

Für die Wirkungsanalyse werden die Befragungsdaten mit umfangreichen Prozessdaten der BA über die Arbeitslosen zusammengespielt, die von den befragten Vermittlern zum Zeitpunkt der Befragung betreut wurden. Dies erfolgt für Deutschland erstmalig; bislang war unbekannt, ob und über welche Zeitintervalle eine solche Zuordnung möglich ist.

Als Prozessdaten nutzen wir die Integrierten Erwerbsbiografien der BA (IEB, siehe Oberschachtsiek et al. 2009), die Daten aus mehreren Fachverfahren enthalten (Beschäftigtenstatistik, Arbeitsuchenden-Statistik, Leistungsempfänger- und Maßnahmehistorik). Um zusätzliche Informationen zu gewinnen und eine höhere Aktualität zu erreichen, greifen wir auch direkt auf Originaldaten aus den Fachverfahren zurück. Diese Daten enthalten tagesgenaue Informationen zu Beschäftigungsverhältnissen, Episoden der Arbeitslosigkeit oder der Arbeitsuche, Bezugszeiten von Arbeitslosengeld bzw. Arbeitslosengeld II sowie die Teilnahme an Programmen der aktiven Arbeitsmarktpolitik. Zudem sind soziodemografische Informationen wie Geschlecht, Gesundheit, Familienstand sowie schulische und Berufsbildung verfügbar. Damit lassen sich standardisierte Erwerbsbiografien der Arbeitslosen über die letzten zehn Jahre konstruieren. Hierzu werden die Informationen über die Erwerbszustände (Beschäftigung, Arbeitslosigkeit, Teilnahme an Maßnahmen sowie Zeiten ohne Nachweis) konsolidiert und die tagesgenauen Erwerbszustände $\mathrm{zu}$ halbmonatlichen Angaben aggregiert. Anschließend werden die halbmonatlichen Angaben über größere Zeitintervalle zusammengefasst und daraus Anteilswerte der Dauern der verschiedenen Erwerbsstatus an diesen Zeitintervallen berechnet. Die Berücksichtigung detaillierter Erwerbsbiografien ist nach Lechner und Wunsch (2013) eine zentrale Voraussetzung für die Validität des verwendeten Matching-Verfahrens. Neben den Erwerbsbiografien

\footnotetext{
${ }^{11}$ Dabei zeigte sich, dass Männer in der verwendeten Stichprobe deutlich überrepräsentiert sind (50,7 versus 30,7\%). Ferner sind Personen mit einem befristeten Arbeitsvertrag in der Stichprobe unterrepräsentiert (14,5 versus $20,8 \%)$ sowie Ältere ab 50 Jahren überrepräsentiert $(29,0$ versus $23,1 \%)$.
}

und soziodemografischen Merkmalen der Arbeitslosen wird der Kalenderzeitpunkt des Eintritts in die Arbeitslosigkeit als zeitinvariante Kovariate berücksichtigt. ${ }^{12}$

Die Zusammenspielung setzt die Zustimmung des jeweiligen Vermittlers voraus, die in 85 Fällen erteilt wurde. Diese Einschränkung führt dazu, dass $46 \%$ der Nettostichprobe von 185 Vermittlern für die Wirkungsanalyse genutzt werden können. Für die Wirkungsanalyse tatsächlich nutzbar sind Angaben von 69 Vermittlern. Um festzustellen, von welchen Determinanten die Zustimmung zur Verknüpfung beeinflusst wird, wurde eine Selektivitätsanalyse durchgeführt. Dabei gingen ausgewählte Strategien der Vermittler und soziodemographische Merkmale als erklärende Variablen ein. Es zeigte sich, dass Männer signifikant häufiger der Verknüpfung zustimmten. Ferner fand sich schwach signifikante Evidenz dafür, dass Vermittler mit einem stärker standardisierten Vorgehen ihr Einverständnis eher gaben als andere. ${ }^{13}$ Die übrigen Strategievariablen waren insignifikant, so dass es keine Hinweise darauf gibt, dass Vermittler mit erfolgreichen Strategien eher der Verknüpfung zugestimmt haben.

Technisch erfolgt das Zusammenspielen über einen internen Datenaufbereitungsprozess der BA, das „IT-Verfahren Datenbasis OpeRative Auswertungen" (DORA), mit dem Auswertungen aus dem Kundenbetreuungsprogramm „VerBIS“ auf Datensatzebene vorgenommen werden können. Ziel ist es dabei, alle Personen zu identifizieren, die von den befragten und mit einer Zuspielung einverstandenen Vermittlern betreut wurden. Die Abfrage wurde am 28. Januar 2010 durch das IT-Systemhaus der BA realisiert. Einzelheiten dazu sind ebenfalls dem erwähnten Projektbericht zu entnehmen (Boockmann et al. 2013).

Um Repräsentativität hinsichtlich der Zugänge in die Arbeitslosigkeit zu erzielen und eine Längsschnittverzerrung zu vermeiden, wird eine Zugangsstichprobe verwendet. Hierzu werden alle Arbeitslosen in die Stichprobe einbezogen, die im Zeitraum vom 12. Mai 2008 bis zum 28. Januar 2010 in die Arbeitslosigkeit zugingen und innerhalb dieser Zeit von einem der befragten Vermittler, die ihre Zustimmung zur Verknüpfung gegeben hatten, betreut wurden. Zusätzlich beschränken wir die Analyse auf diejenigen Verläufe, bei denen der Betreuungsbeginn durch den befragten Vermittler innerhalb von 60 Tagen nach Beginn der Arbeitslosigkeit einsetzte, um zu vermeiden, dass ein später Betreuungsbeginn durch diesen Vermittler fälschli-

\footnotetext{
${ }^{12}$ Tabelle 6 im Anhang enthält nähere Informationen zu den verwendeten Variablen. In Abschnitt 4.2 werden weitere Details beschrieben.

${ }^{13}$ Während $55 \%$ der Männer im SGB-III-Bereich ihre Zustimmung gaben, stimmten lediglich $35 \%$ der Frauen der Verknüpfung zu. Die Ergebnisse der multivariaten Selektivitätsanalysen sind auf Anfrage von den Verfassern erhältlich.
} 
cherweise für zuvor gemessene Integrationsergebnisse für die betreuten Personen verantwortlich gemacht wird. ${ }^{14}$

Die Untersuchung wird außerdem auf Arbeitslose im Alter von 25 bis unter 55 Jahre begrenzt, weil für spezielle Gruppen wie Jugendliche oftmals spezielle Vermittler zuständig sind. Insgesamt stehen damit 22.746 Arbeitslosigkeitsepisoden von 20.944 Personen zur Verfügung. Die Anzahl der einem Vermittler zugewiesenen und in unseren Auswertungen einbezogenen Betreuungsfälle reicht von 46 bis 783, mit einem Durchschnitt von 330 betreuten Personen. ${ }^{15}$

\subsection{Deskriptive Ergebnisse zum Verlauf der Ergebnisvariablen}

Aus den Prozessdaten werden zwei Ergebnisvariablen konstruiert: zum einen der erstmalige Abgang aus der Arbeitslosigkeit, zum anderen die erstmalige Aufnahme eines sozialversicherungspflichtigen Beschäftigungsverhältnisses nach der Betreuung durch den befragten Vermittler. Wir unterscheiden zwischen diesen beiden Ergebnisvariablen, da der Abgang aus Arbeitslosigkeit nicht mit der Aufnahme einer Beschäftigung einhergehen muss. So ist ein Abgang aus Arbeitslosigkeit auch bei einer Abmeldung aus Arbeitslosigkeit in Nichterwerbstätigkeit zu verzeichnen. Gleiches gilt beim Eintritt in eine arbeitsmarktpolitische Maßnahme. ${ }^{16}$ Die Berücksichtigung von zwei verschiedenen Ergebnisvariablen erlaubt nicht nur eine vergleichende Interpretation der Ergebnisse, sondern trägt auch den gesetzlichen Zielen der Arbeitsförderung Rechnung. ${ }^{17}$

Verwendet werden die kumulierten Wahrscheinlichkeiten, den jeweiligen Zustand bis zum betrachteten Zeitpunkt verlassen zu haben, also eins minus die Überlebensfunktion für den Verbleib in Arbeitslosigkeit. Abbildung 1 zeigt die kumulierten Wahrscheinlichkeiten des Abgangs aus der Arbeitslosigkeit und des Übergangs in Beschäftigung in Abhängigkeit von der Dauer der Arbeitslosigkeit, die ab

\footnotetext{
${ }^{14}$ In einer Robustheitsanalyse wurde dieses Intervall auf 30 Tage reduziert. In einer weiteren Robustheitsanalyse wurde die Stichprobe auf Episoden der Arbeitslosigkeit begrenzt, deren Beginn in einem zeitlichen Abstand von der Befragung von nicht mehr als einem halben Jahr liegen, da sich die Strategien der Vermittler im Zeitablauf ändern können. Wir gehen bei der Interpretation der Treatment-Effekte in Abschnitt 5 auf die Ergebnisse dieser Robustheitsanalysen ein.

${ }^{15}$ Bezieht man die für diese Analyse ausgeschlossenen Personen mit ein, so ergeben sich für unseren Datensatz 61 bis 947 einer Vermittlungsfachkraft zugewiesene Arbeitslose und ein Durchschnitt von 428 betreuten Personen.

${ }^{16}$ Der Zugang in arbeitsmarktpolitische Maßnahmen kann auch als eigene Ergebnisvariable verwendet werden. Dabei zeigt sich, dass es sinnvoll ist, zwischen unterschiedlichen Kategorien von Maßnahmen zu unterscheiden (siehe dazu Boockmann und Neumann 2012).

${ }^{17}$ In $\S 1$ SGB III werden sowohl ein hoher Beschäftigungsstand als auch eine Verkürzung der Dauer der Arbeitslosigkeit als Ziele genannt.
}

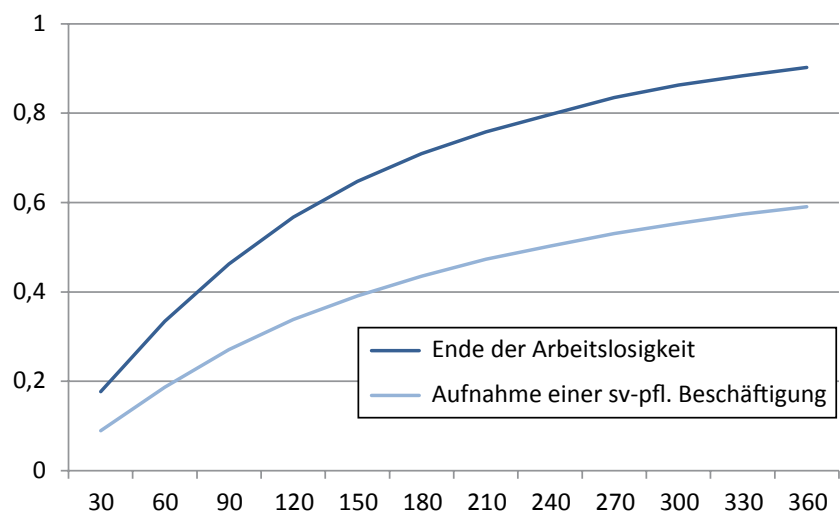

Abb. 1 Abgang aus Arbeitslosigkeit und Aufnahme einer sozialversicherungspflichtigen Beschäftigung. Auf der vertikalen Achse ist die kumulierte Wahrscheinlichkeit eines Übergangs abgetragen. Sie entspricht dem Anteil der Personen, die bis zur jeweiligen Dauer der Arbeitslosigkeit ihre Arbeitslosigkeit beendet oder eine Beschäftigung aufgenommen hatten. Auf der horizontalen Achse ist die Dauer der Arbeitslosigkeit in Tagen abgetragen. (Quelle: IAB-Befragung von Vermittlern und Fallmanagern im Jahr 2009. Administrative Daten der BA. Eigene Berechnungen)

dem in den Daten gefundenen Zugangsdatum gemessen wird. Als Abgang aus der Arbeitslosigkeit wird das Ende einer Arbeitslosigkeits-Episode definiert, wobei innerhalb der auf das Ende folgenden 30 Tage keine erneute Episode der Arbeitslosigkeit folgen darf. Die Abbildung zeigt, dass der Abgang aus Arbeitslosigkeit mit einer wesentlich höheren Rate erfolgt als der Übergang in sozialversicherungspflichtige Beschäftigung. Nach 360 Tagen sind bereits 90,2\% der Arbeitslosigkeits-Episoden in den Daten beendet. Zu diesem Zeitpunkt sind jedoch erst 59,1\% der Arbeitslosen in sozialversicherungspflichtige Beschäftigung übergegangen, wobei dies nicht notwendigerweise mit einem Ende der Arbeitslosigkeit einhergeht. Ein großer Teil der Arbeitslosen beendet also die Arbeitslosigkeit, ohne dass die Aufnahme einer Beschäftigung zu beobachten ist. Dabei entwickeln sich beide Ergebnisvariablen über die Zeit nach dem gleichen Muster: es werden mit zunehmender Dauer der Arbeitslosigkeit immer weniger Übergänge pro Zeiteinheit beobachtet. Dies könnte unter anderem darauf zurückzuführen sein, dass der Anteil der arbeitsmarktferneren Personen an den noch nicht Vermittelten mit der Zeit zunimmt. Es könnte aber auch durch einen echten Zeitdauereffekt erklärt werden, der sich beispielsweise dadurch einstellt, dass Personen mit längerer Arbeitslosigkeitsdauer stigmatisiert sind. ${ }^{18}$

Da infolge der Bezugsdauer von Arbeitslosengeld I der Übergang aus dem Rechtskreis des SGB III in den SGB-II-

\footnotetext{
${ }^{18}$ Für eine vertiefte Analyse wären die individuelle Heterogenität und die Zeitdauerabhängigkeit in der Darstellung zu trennen; wir haben hier darauf verzichtet, da dies außerhalb des Fokus unseres Beitrages liegt.
} 
Bereich in der Regel nach 360 Tagen erfolgt,${ }^{19}$ endet die Messung der Ergebnisvariablen mit diesem Zeitpunkt. Danach findet durch den Wechsel des Rechtskreises zwangsläufig ein Wechsel der Betreuungsperson statt. Zwar ist nicht ausgeschlossen, dass die Betreuung durch einen bestimmten SGB-III-Vermittler auch nach dem Wechsel in den Rechtskreis des SGB II noch eine Wirkung zeigt. Primär dürften dann jedoch die Strategien der SGB-II-Vermittler wirksam sein, die die Betreuung der jeweiligen Person übernehmen. Von diesen Vermittlern liegen jedoch im Regelfall keine Befragungsdaten vor.

\section{Verfahren der Wirkungsanalyse}

\subsection{Zuweisung der Arbeitslosen zu Vermittlern}

Für die Wirkungsanalyse ist es von fundamentaler Bedeutung, wie die Arbeitslosen den Vermittlern, die bestimmte Strategien anwenden, zugeteilt werden. Dabei ist zwischen einem systematischen Prozess, bei dem die Zuteilung von bestimmten Eigenschaften abhängt, und einer quasi-zufälligen Zuteilung zu unterscheiden. Bei einer systematischen Zuteilung ist wiederum zu fragen, ob die Eigenschaften, aufgrund derer die Zuteilung vorgenommen wird, in den Daten beobachtet und daher im Schätzverfahren berücksichtigt werden können. Problematisch wäre es, wenn die Gründe für die Zuteilung nicht beobachtet werden. Dies wäre beispielsweise dann der Fall, wenn bestimmte Vermittler sich auf die Vermittlung von Arbeitslosen mit motivationalen Problemen konzentrieren würden oder wenn Vermittler, denen über einen Zeitraum besonders viele Arbeitslose mit motivationalen Problemen zugewiesen werden, daraufhin ihre Vorgehensweise ändern würden. Da motivationale Probleme in den vorliegenden Daten der Kunden nicht zu beobachten sind, könnte nicht berücksichtigt werden, dass Arbeitslose mit solchen Problemen durchschnittlich schlechtere Integrationschancen haben. Stattdessen würden diese fälschlicherweise der Strategie der Vermittler zugerechnet.

Tabelle 1 enthält Informationen darüber, wie die Arbeitsuchenden den Vermittlern in den in die Befragung einbezogenen Agenturen für Arbeit zugeteilt werden. Die Zuweisung basiert in den meisten Fällen auf dem Beruf der Kunden. Die Zugehörigkeit zu einer Berufsgruppe könnte die Anwendung bestimmter Strategien bedingen. Beispielsweise geht ein Vermittler, der Akademiker berät, möglicherweise anders vor als ein Vermittler, der gering Qualifizierte

\footnotetext{
${ }^{19}$ Ausnahmen davon sind Personen mit kurzer Dauer des vorhergehenden versicherungspflichtigen Beschäftigungsverhältnisses und entsprechend kürzerer Dauer des Anspruchs auf Arbeitslosengeld sowie ältere Arbeitslose ab 50 Jahren, deren Anspruchsdauer in dem betrachteten Zeitraum bis zu 15 Monate betragen kann.
}

Tab. 1 Kriterien der Zuteilung von Kunden zu Vermittlern. (Quelle: IAB-Befragung von Vermittlern und Fallmanagern im Jahr 2009. Eigene Berechnungen)

\begin{tabular}{lll}
\hline Kriterium & $\begin{array}{l}\text { Anteil der } \\
\text { Kunden }\end{array}$ & $\begin{array}{l}\text { Anteil der } \\
\text { Vermittler }\end{array}$ \\
\hline Nach Berufsgruppen & 79,5 & 81,2 \\
Nach den Anfangsbuchstaben der & 41,0 & 34,8 \\
Familiennamen & & \\
Nach räumlicher Zugehörigkeit & 28,5 & 23,2 \\
Nach Auslastung des & 9,5 & 8,7 \\
Vermittlers/der Vermittlerin & & \\
Andere & 7,2 & 10,1 \\
\hline Mehrfachnennungen waren zulässig. Alle Angaben in Prozent
\end{tabular}

betreut. Insofern wäre die Zuweisung zu Strategien nicht wie in einer experimentellen Situation zufällig, sondern von den Eigenschaften der Betreuten beeinflusst. Allerdings lässt sich dies in der Wirkungsanalyse berücksichtigen, indem nur Angehörige desselben Berufs miteinander verglichen werden.

Auch die anderen Zuweisungsmechanismen können nicht als zufällig betrachtet werden. Der Wohnort der Betreuten könnte bei entsprechender räumlicher Segregation leicht mit soziodemografischen Merkmalen zusammenhängen. Der Anfangsbuchstabe des Namens korreliert mit der ethnischen Herkunft der Person (so sind bestimmte Anfangsbuchstaben wie Y in Deutschland selten). Für diese nicht-zufälligen Zuweisungsprozesse zu bestimmten Vermittlern muss kontrolliert werden. Dies ist mit dem vorhandenen Datensatz beispielsweise dadurch möglich, dass Informationen über die Staatsangehörigkeit vorliegen. ${ }^{20}$

Insgesamt lässt sich argumentieren, dass die in Wirkungsanalysen stets zu berücksichtigende Selektion in das Treatment in unserer Anwendung ein lösbares Problem ist, da sie nicht - wie in weiten Bereichen der Evaluation arbeitsmarktpolitischer Maßnahmen - von unbeobachteten Faktoren wie Motivation und Kompetenzen getrieben wird, sondern von prinzipiell beobachtbaren Eigenschaften. Die verbliebenen Unterschiede in der Zuteilung, die nicht durch die genannten grundsätzlich beobachtbaren Merkmale erklärt werden können, können daher als zufällig angesehen werden. Sie könnten beispielsweise durch die vorübergehende krankheits- oder urlaubsbedingte Abwesenheit eines Vermittlers und die (vorübergehende) Zuteilung der entsprechenden Kunden zu anderen Vermittlern bedingt sein.

\subsection{Matching-Verfahren zur Kontrolle von Selektivität}

Um den Einfluss der Strategien und Einstellungen auf die Arbeitsmarktergebnisse zu schätzen, wenden wir das Propensity-Score-Matching-Verfahren an (siehe z. B. Abadie

\footnotetext{
${ }^{20}$ Hierbei ist allerdings zu berücksichtigen, dass die Staatsangehörigkeit die ethnische Herkunft nur grob approximiert.
} 
und Imbens 2006; Heckman et al. 1999). Das Matching-Verfahren wird verwendet, um das Grundproblem der Evaluationsforschung zu lösen. Dieses besteht darin, dass dieselbe Person nicht in zwei sich einander ausschließenden Situationen gleichzeitig beobachtet werden kann. Beispielsweise kann eine Person nicht durch einen Vermittler mit einer bestimmten Einstellung oder Handlungsweise betreut und gleichzeitig nicht betreut werden. Die kontrafaktische Situation kann also nie beobachtet werden. Das Grundprinzip des Matching-Verfahrens besteht darin, ex post eine Kontrollgruppe zu finden, deren Nichtzugang in das Treatment als zufällig angesehen werden kann. Dies wird beim hier verwendeten Propensity Score Matching dadurch erreicht, dass die Personen in der Treatment- und Kontrollgruppe jeweils dieselbe Wahrscheinlichkeit für den Zugang zum Treatment aufweisen, so dass es als zufällig gelten kann, ob sie tatsächlich das Treatment bekommen haben.

Dabei wird in einem ersten Schritt für jede Person die Wahrscheinlichkeit vorhergesagt, von einem Vermittler betreut $\mathrm{zu}$ werden, der die jeweils betrachtete Einstellung oder Handlungsweise aufweist. Auf dieser Basis werden Personen verglichen, die (nahezu) dieselben Wahrscheinlichkeiten für die betrachtete Art der Betreuung haben.

In die hierzu vorgenommenen Schätzungen, die durch Probit-Modelle vorgenommen werden, fließen das Geschlecht, das Alter, die schulische und Berufsausbildung, gesundheitliche Einschränkungen sowie der Schwerbehindertenstatus, die Staatsangehörigkeit und der Familienstand als unabhängige Variablen ein. Zusätzlich werden über Dummy-Variablen der Beruf und die Agentur berücksichtigt. Aus den Beschäftigungs-, Maßnahme- und LeistungsHistoriken lassen sich weiter die Erwerbsbiografien der Individuen innerhalb der letzten zehn Jahre darstellen. Dazu wird der jeweilige Zeitanteil in den Zuständen Beschäftigung, Arbeitslosigkeit, Nichterwerbstätigkeit und Maßnahmeteilnahme berechnet. In die Schätzungen gehen sie als binäre Variablen ein, die angeben, ob die Zeitanteile unterhalb des ersten oder oberhalb des dritten Quartils aller Personen in der Stichprobe liegen. Ferner werden die Variablen mit einem Altersdummy interagiert, um zu berücksichtigen, dass jüngere Personen möglicherweise noch keine zehnjährige Erwerbsbiografie haben..$^{21}$ Die Schätzergebnisse der Probit-Modelle für die insgesamt sieben Treatment-Variablen ${ }^{22}$ zeigen, dass die Koeffizienten für die meisten Variablen mit Ausnahme des Berufs, der Ausbildungsvariablen und der Agenturen insignifikant sind, was bestätigt, dass die

\footnotetext{
${ }^{21}$ Tabelle 6 im Anhang listet die verwendeten Variablen und ihre Mittelwerte auf. Ergänzend wurden Robustheitsanalysen vorgenommen, in denen Eigenschaften der Vermittler - Geschlecht, Erfahrung und Ausbildung - in die Schätzungen eingingen.

${ }^{22}$ Die Beschreibung der Treatment-Variablen ist im nächsten Abschnitt zu finden. Die Ergebnisse der Probitschätzungen sind in Tabelle 7 im Anhang enthalten.
}

Zuweisung zu den Vermittlern überwiegend auf der Basis des Berufs und nicht anhand anderer individueller Merkmale vorgenommen wird. Gelegentlich finden sich auch signifikante Effekte für die Staatsangehörigkeit, was an der Zuweisung nach Anfangsbuchstaben des Alphabets oder des Wohngebiets liegen könnte, sowie für den Familienstand.

In der zweiten Stufe wird auf der Basis der vorhergesagten Wahrscheinlichkeiten das Matching in Form eines Kernel-Matchings vorgenommen. Ausgeschlossen werden Beobachtungen außerhalb des ,common support“, also des Bereichs der Verteilungen des Propensity Scores, die sich überschneiden. Die Standardfehler werden durch ein „block bootstrap"-Verfahren geschätzt. ${ }^{23}$ Dadurch wird berücksichtigt, dass das Treatment auf der Ebene der 69 Vermittler und nicht der einzelnen betreuten Person variiert.

Bei der Spezifikation des Propensity Scores werden zwei unterschiedliche Varianten verwendet:

1. Es werden nur Personen in derselben Agentur verglichen. Damit wird berücksichtigt, dass die Arbeitsmarktsituation und die Herangehensweise der Agenturen sich in den zehn Agenturbezirken so stark unterscheiden können, dass man keine Vergleichspaare über die Grenzen der Agenturbezirke hinweg bilden sollte.

2. Es werden zusätzlich nur Personen verglichen, die derselben Berufsgruppe angehören. Damit wird berücksichtigt, dass die Zuweisung - abgesehen vom Beruf - bei den meisten Agenturen zufällig ist, wie in Abschnitt 4.1 dargelegt.

Zwar ist die erwartete Verzerrung in den geschätzten Treatment-Effekten bei der zweiten Variante geringer als bei der ersten. Jedoch könnte es sein, dass sich die Qualität der Übereinstimmung der Merkmale von Treatment- und Kontrollgruppe in Variante zwei gegenüber Variante eins verschlechtert, weil weniger potenzielle Matching-Partner zur Verfügung stehen.

Tabelle 2 zeigt für die beiden Varianten die Qualität des Matchings. Hierzu wurden die standardisierten Abweichungen jeweils zwischen der Treatment- und der gematchten Kontrollgruppe für alle verwendeten Kontrollvariablen berechnet. Dargestellt ist der Medianwert über alle Kontrollvariablen. Als Faustregel wird häufig eine standardisierte Abweichung von 4 als tolerabel angesehen (Caliendo und Kopeinig 2008). Nach dem Matching liegt der Median der standardisierten Abweichung teilweise deutlich unterhalb dieser Marke. Dabei zeigen sich keine erheblichen Unterschiede zwischen den beiden Varianten. Deshalb werden im Folgenden die Ergebnisse von Variante zwei berichtet, da in diesem geringere Verzerrungen zu erwarten sind. Die Unterschiede in den geschätzten Treatment-Effekten zwischen beiden Ansätzen sind jedoch gering.

\footnotetext{
${ }^{23}$ Dabei werden 200 Replikationen verwendet.
} 
Tab. 2 Tests auf die Qualität des Matching: Standardisierte Abweichungen (Median) vor und nach dem Matching. (Quelle: IAB-Befragung von Vermittlern und Fallmanagern im Jahr 2009. Administrative Daten der BA. Eigene Berechnungen)

\begin{tabular}{llllll}
\hline Treatment-Variable & Spezifikation 1 & & & Spezifikation 2 \\
\cline { 2 - 3 } \cline { 5 - 6 } & Vor Matching & Nach Matching & & Vor Matching & Nach Matching \\
\hline Kontaktdichte & 5,13 & 1,54 & 5,13 & 2,54 \\
Schnelle Wiedereingliederung & 5,98 & 1,51 & 5,98 & 1,23 \\
Sperrzeiten nicht nur im Notfall & 5,16 & 1,68 & 5,16 & 1,94 \\
Eingehen auf Wünsche des Arbeitslosen & 4,28 & 2,71 & 4,28 & 1,45 \\
Individuelle Verantwortung für Arbeitslosigkeit & 4,24 & 1,54 & 4,24 & 1,55 \\
Bedeutung ermessenslenkender Weisungen & 7,75 & 1,73 & 7,75 & 0,90 \\
Entscheidungsfreiheit & 7,60 & 2,45 & 7,60 & 2,21 \\
\hline
\end{tabular}

Dargestellt sind die standardisierten Abweichungen

\section{Ergebnisse der Wirkungsanalyse}

Im Folgenden stellen wir die Ergebnisse für sieben einzelne Gegenstände der Befragung vor. Entsprechend den in Abschnitt 2 dargestellten Aspekten des Vermittlerhandelns untersuchen wir folgende Treatments (siehe zur genauen Definition auch Tab. 3):

- Die Intensität der Vermittlung wird durch die Kontaktdichte erfasst. Im Fragebogen wird dabei nach SGB-IIIKundengruppen differenziert; als Treatment-Variable wird jedoch ein zusammenfassendes Maß gebildet, das angibt, ob mit den Kunden aller Kundengruppen mindestens alle zwei Monate ein persönliches Gespräch geführt wird. ${ }^{24}$

- Weiterhin wird eine Variable untersucht, die die Ziele der Vermittlung erfasst. Dabei wird unterschieden, ob sich die Arbeitsförderung als allererstes auf eine grundsätzlich schnelle Wiedereingliederung, auf eine nachhaltige Wiedereingliederung oder auf die Stabilisierung der persönlichen Situation konzentrieren sollte.

- Als grundlegende Einstellung wird die Zustimmung zu der Aussage untersucht, dass Arbeitslosigkeit individuell und nicht gesellschaftlich bedingt ist.

- Die Wirkung finanzieller Sanktionen wird durch eine Aussage zur Nutzung von Sperrzeiten analysiert.

- Der Grad der Kooperation mit den Arbeitslosen wird ähnlich wie in Behncke et al. (2010a) - durch die Bereitschaft erfasst, bei der Maßnahmevergabe auf Wünsche der Arbeitslosen einzugehen.

- Die Handlungsspielräume werden durch die Bedeutung von Weisungen bei der Maßnahmevergabe konkretisiert.

- Um festzustellen, in welchem Umfang die Fachkräfte sich Orientierung durch Regeln wünschen, wird eine Aussage über die Wichtigkeit von Normen oder Entscheidungsfreiheit herangezogen.

\footnotetext{
${ }^{24}$ Mit Ausnahme der Betreuungskunden, bei denen bereits ein Gespräch mindestens alle vier Monate als hohe Kontaktdichte angesehen wird. Die Zusammenfassung der Indikatoren für die Kundengruppen geschieht auch deshalb, weil in den Prozessdaten keine Information über die Zugehörigkeit der Kunden zu Kundengruppen enthalten ist.
}

Die Zustimmung oder Ablehnung zu den genannten Aussagen wird auf einer fünfstufigen Skala erhoben; in der Wirkungsanalyse wird diese Information zu binären Variablen verdichtet. Inhaltlich werden sowohl Strategien - beispielsweise die Vergabe von Sanktionen oder die Kontaktdichte - als auch Einstellungen - beispielsweise zu den Zielen der Vermittlung oder zur Verursachung von Arbeitslosigkeit erhoben. Wir gehen davon aus, dass es sich bei diesen nicht lediglich um Meinungsäußerungen handelt sondern um die Basis für das berufliche Selbstverständnis. Damit verbinden wir, dass sie mindestens die Kommunikation mit dem Betreuten bestimmen und sich auch in bestimmten Strategien (z. B. einer höheren Bereitschaft, Sanktionen zu ergreifen oder Maßnahmen zu vergeben) niederschlagen. Mit der Abfrage der Einstellungen wird insofern die Gesamtheit der vom Vermittler ausgehenden Signale an die Arbeitslosen erfasst, die jeweils nicht einzeln beobachtet werden können. Dass Einstellungen in Signale und Handlungen übersetzt werden, muss eine Annahme bleiben, da auf der Basis der verfügbaren Daten eine Überprüfung nicht möglich ist.

Die verschiedenen Einstellungen und Strategiebereiche müssen nicht unabhängig voneinander sein. Tabelle 4 zeigt die Korrelationen zwischen den einzelnen Treatment-Variablen. Vermittler, die angeben, eine grundsätzlich schnelle Wiedereingliederung anzustreben, sehen die Schuld für Arbeitslosigkeit auch häufig bei den Arbeitslosen selbst. Vermittler, die Sanktionen nach eigenen Angaben nicht nur im Notfall einsetzen, gehen auch weniger häufig auf Wünsche der Arbeitslosen bei der Maßnahmevergabe ein. Zudem korrelieren die Aussagen zur Bedeutung interner Weisungen und zur Entscheidungsfreiheit negativ miteinander. Da die Treatment-Variablen nur einzeln in die Analyse eingehen, muss bei der Interpretation der Ergebnisse berücksichtigt werden, wie die Ergebnisse durch die Korrelation mit jeweils nicht berücksichtigten Treatment-Variablen beeinflusst werden könnten.

Abbildung 2 zeigt die Ergebnisse der Wirkungsanalyse. In der linken Spalte finden sich die Ergebnisse für den Abgang aus der Arbeitslosigkeit, in der rechten Spalte die für die Aufnahme einer sozialversicherungspflichtigen Beschäftigung. Ausgewiesen sind jeweils die Effekte auf die 
Tab. 3 Einzelne berücksichtigte Strategien und Einstellungen. (Quelle: IAB-Befragung von Vermittlern und Fallmanagern im Jahr 2009. Eigene Berechnungen)

Treatment-Variable

Fragebogenformulierung und Definition

Relative Häufigkeit

Kontaktdichte

Wahl der Antwortmöglichkeiten ,einmal im Monat oder öfter“ oder ,,alle ein bis zwei Monte

0,31

auf die Frage „Wie häufig sehen Sie - alles in allem - Ihre Kunden/Kundinnen im Alter

zwischen 25 und 58 Jahren zu persönlichen Gesprächen im Durchschnitt?“ in Bezug auf jede

der drei Kundengruppen „Marktkunde“, „Beratungskunde Aktivieren“ und „Beratungskunde

Fördern“. Bezüglich der Kundengruppe Betreuungskunde kann zusätzlich auch die Option ,,alle drei bis vier Monate" gewählt werden

Schnelle Wahl der Antwortmöglichkeit ,grundsätzlich schnelle Wiedereingliederung von Arbeitslosen“

Wiedereingliederung anstelle von „nachhaltige Eingliederung“ oder „Stabilisierung der persönlichen Situation“ auf die Frage „Auf welche Strategie sollte man die Mittel der Arbeitsförderung Ihrer Meinung nach als allererstes konzentrieren?"

Individuelle Verantwortung für Einordnung bei den Werten 4 und 5 auf einer Skala von 1: „Arbeitslosigkeit ist heute ein gesellschaftlicher Tatbestand und einfach persönliches Schicksal, an dem der/die Einzelne keine eigene Schuld trägt“ bis zu 5: ,Jede/r Arbeitslose ist letztlich selbst verantwortlich für seine Situation und kann etwas daran ändern.“

Arbeitslosigkeit Wahl der Option "Ich stimme gar nicht zu“ auf die Aussage „Sperrzeiten setze ich nur im Not-

Sperrzeiten nicht nur fall durch, um das Verhältnis zum Kunden/zur Kundin nicht zu belasten“"

im Notfall

Eingehen auf Wünsche Wahl der Option „Wunsch der Stellensuchenden“ bei mindestens drei von fünf Maßnahmendes Arbeitslosen gruppen auf die Frage „Aus welchen Gründen setzen Sie die folgenden Maßnahmen vorwiegend ein?"

Bedeutung ermessens- Wahl der Optionen „hoch“ oder „sehr hoch“ bei der Frage „Welchen Stellenwert haben bei der lenkender Weisungen Ihrer Entscheidung für die eingesetzten Instrumente sonstige interne Weisungen?“

Entscheidungsfreiheit Einordnung bei den Werten 4 und 5 auf einer Skala von 1: „Regeln und Handlungsanweisungen sind unabdingbar notwendige Grundlagen für meine Arbeit“ bis zu 5: „Um meine tägliche Arbeit erfolgreich machen zu können, brauche ich völlige Entscheidungsfreiheit"

Die relative Häufigkeit ist der Anteil der Kunden, die von Vermittlern betreut wurden, die der jeweiligen Aussage der Treatment-Variablen zugestimmt haben

Tab. 4 Korrelation der in der Wirkungsanalyse verwendeten Treatmentvariablen. (Quelle: IAB-Befragung von Vermittlern und Fallmanagern im Jahr 2009. Eigene Berechnungen)

\begin{tabular}{|c|c|c|c|c|c|c|c|}
\hline Treatment-Variablen & $\begin{array}{l}\text { Hohe } \\
\text { Kontaktdichte }\end{array}$ & $\begin{array}{l}\text { Grundsätzlich } \\
\text { schnelle Wieder- } \\
\text { eingliederung }\end{array}$ & $\begin{array}{l}\text { Individuelle Ver- } \\
\text { antwortung für } \\
\text { Arbeitslosigkeit }\end{array}$ & $\begin{array}{l}\text { Sperrzeiten } \\
\text { nicht nur im } \\
\text { Notfall }\end{array}$ & $\begin{array}{l}\text { Eingehen auf } \\
\text { Wünsche der } \\
\text { Arbeitslosen }\end{array}$ & $\begin{array}{l}\text { Bedeutung von } \\
\text { sonstigen inter- } \\
\text { nen Weisungen }\end{array}$ & $\begin{array}{l}\text { Entschei- } \\
\text { dungsfreiheit }\end{array}$ \\
\hline \multicolumn{8}{|l|}{ Hohe Kontaktdichte } \\
\hline $\begin{array}{l}\text { Grundsätzlich schnelle } \\
\text { Wiedereingliederung }\end{array}$ & 0,044 & & & & & & \\
\hline $\begin{array}{l}\text { Individuelle Verantwor- } \\
\text { tung für Arbeitslosigkeit }\end{array}$ & $-0,008$ & 0,295 & & & & & \\
\hline $\begin{array}{l}\text { Sperrzeiten nicht nur im } \\
\text { Notfall }\end{array}$ & 0,046 & $-0,131$ & 0,156 & & & & \\
\hline $\begin{array}{l}\text { Eingehen auf Wünsche } \\
\text { der Arbeitslosen }\end{array}$ & 0,044 & 0,093 & 0,007 & $-0,260$ & & & \\
\hline $\begin{array}{l}\text { Bedeutung von sonsti- } \\
\text { gen internen Weisungen }\end{array}$ & 0,066 & $-0,120$ & $-0,171$ & $-0,100$ & $-0,055$ & & \\
\hline Entscheidungsfreiheit & $-0,054$ & $-0,180$ & 0,136 & 0,038 & $-0,101$ & $-0,312$ & \\
\hline
\end{tabular}

entsprechenden Übergangswahrscheinlichkeiten in Prozentpunkten. Die Zeitachse gibt die Dauer der Arbeitslosigkeit in Tagen an; die Messung endet ein Jahr nach Eintritt in die Arbeitslosigkeit. Die gestrichelten, helleren Linien entsprechen den Ober- und Untergrenzen des 95-\%-Konfidenzintervalls für den geschätzten Effekt; bei der Interpretation der Ergebnisse beziehen wir uns - sofern im Text nicht anders erwähnt - auf ein Signifikanzniveau von fünf Prozent. ${ }^{25}$

${ }^{25}$ Lesebeispiel: Ein Wert von ca. 0,02 für das Treatment „Hohe Kontaktdichte" nach 60 Tagen in der linken Spalte bedeutet, dass sich die
Auf der vertikalen Achse ist der Treatment-Effekt in Prozentpunkten abgetragen, auf der horizontalen Achse die Dauer der Arbeitslosigkeit in Tagen.

Die Ergebnisse zeigen zunächst, dass eine höhere Kontakthäufigkeit tendenziell zu einer erhöhten Abgangswahrscheinlichkeit aus der Arbeitslosigkeit führt, wobei der Effekt nur für einen relativ kleinen Zeitraum (120 bis 150

Wahrscheinlichkeit eines Abgangs aus der Arbeitslosigkeit durch hohe Kontaktdichte gegenüber der kontrafaktischen Situation geringer Kontaktdichte um ungefähr zwei Prozentpunkte erhöht. 
Ergebnisvariable: Ende der Arbeitslosigkeit

Ergebnisvariable: Aufnahme einer svpfl. Beschäftigung

Hohe Kontaktdichte, $\mathrm{N}=6.778$ (Treatmentgruppe), $\mathrm{N}=13.508$ (Kontrollgruppe nach Matching)
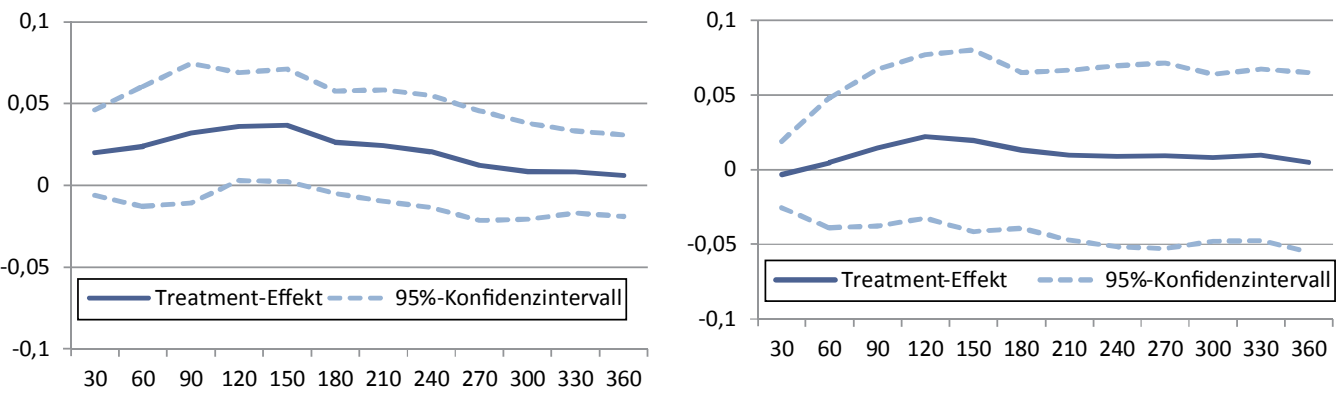

Grundsätzlich schnelle Wiedereingliederung, $\mathrm{N}=9.313$ (Treatmentgruppe), $\mathrm{N}=12.547$ (Kontrollgruppe nach Matching)
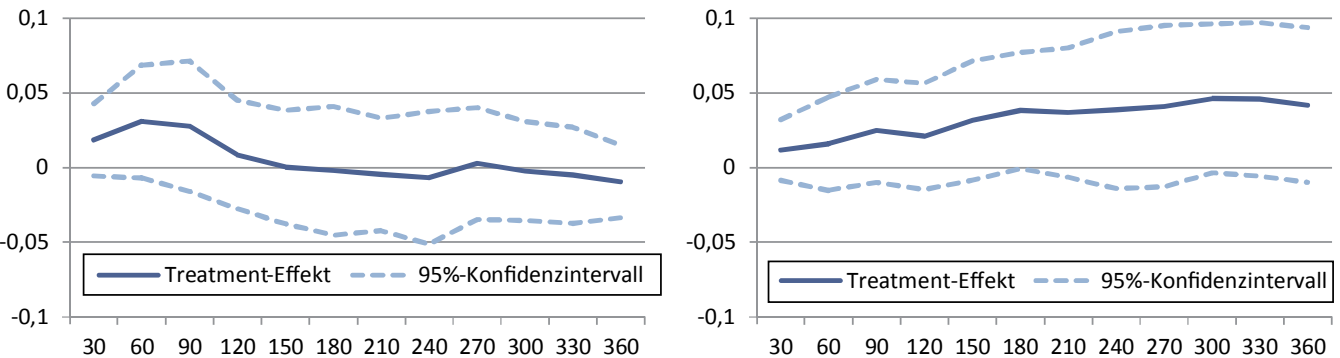

Individuelle Verantwortung für Arbeitslosigkeit, $\mathrm{N}=4.672$ (Treatmentgruppe), $\mathrm{N}=16.007$ (Kontrollgruppe nach Matching)
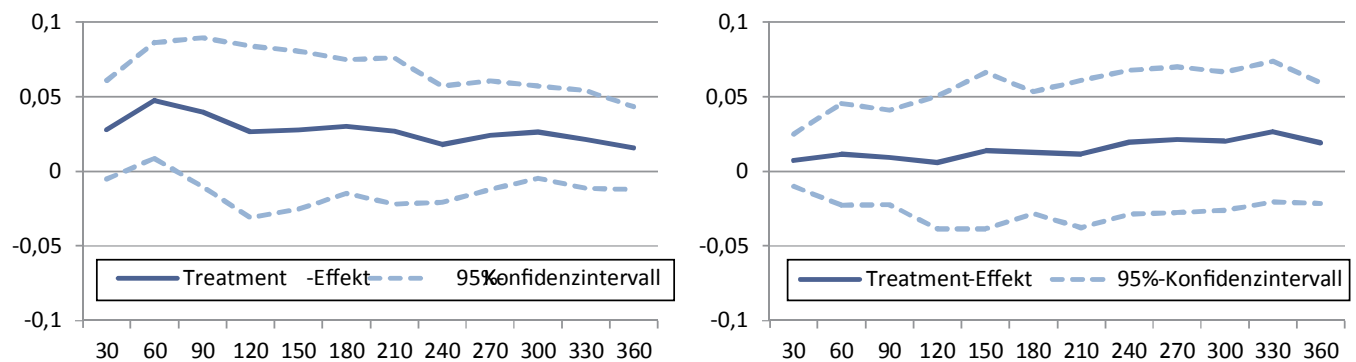

Sperrzeiten nicht nur im Notfall, $\mathrm{N}=9.424$ (Treatmentgruppe), $\mathrm{N}=9.863$ (Kontrollgruppe nach Matching)
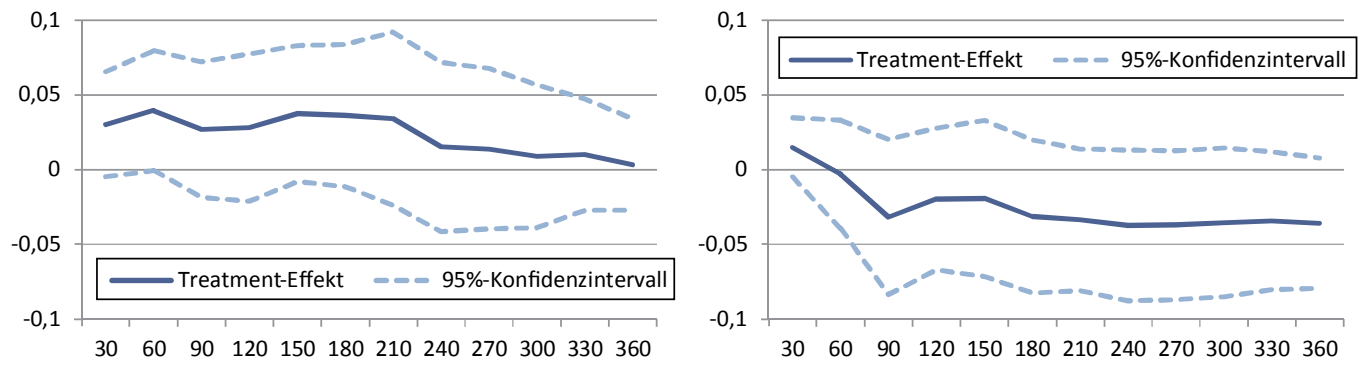

Eingehen auf Wünsche der Arbeitslosen, $\mathrm{N}=7.404$ (Treatmentgruppe), $\mathrm{N}=12.905$ (Kontrollgruppe nach Matching)
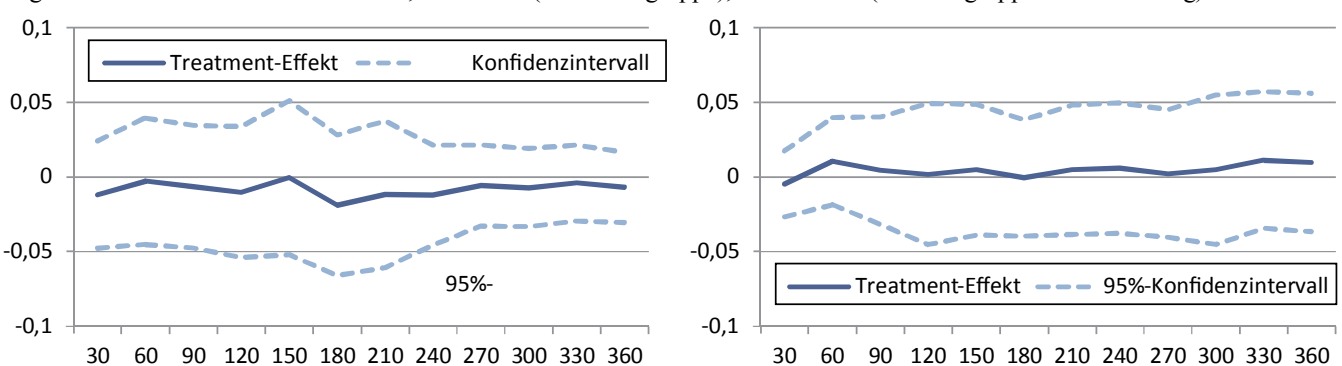

Abb. 2 Geschätzte Treatment-Effekte einzelner Strategien. (Quelle: IAB-Befragung von Vermittlern und Fallmanagern im Jahr 2009. Administrative Daten der BA. Eigene Berechnungen) 
Ergebnisvariable: Ende der Arbeitslosigkeit

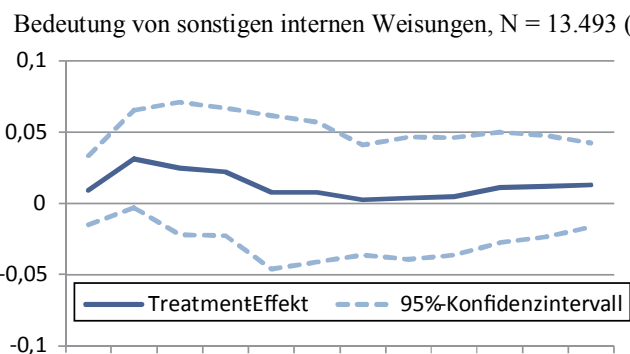

$30 \quad 6090120150180210240270300330360$

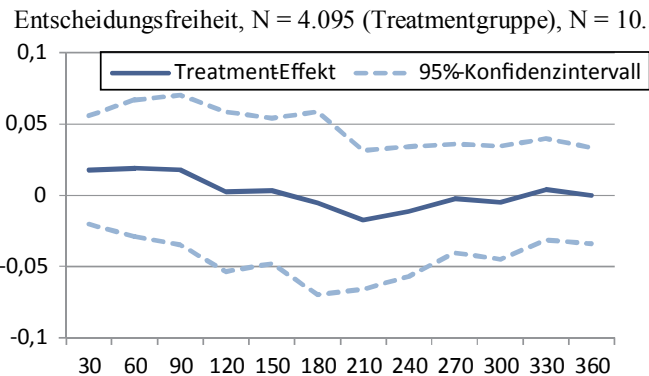

Abb. 2 (Fortsetzung)

Tage nach Beginn der Arbeitslosigkeit) signifikant ist und sich nach diesem Zeitpunkt in der Größenordnung deutlich abschwächt. In der Wirkungsrichtung bestätigen diese Ergebnisse frühere Studien, wonach die Kontaktdichte zwischen Arbeitslosen und Vermittlern die Integrationswahrscheinlichkeit positiv beeinflusst (beispielsweise van den Berg et al. 2012), insbesondere zu Beginn der Arbeitslosigkeit (Wunsch 2013). ${ }^{26}$ Die Ergebnisse für den Übergang in Beschäftigung sind allerdings insignifikant.

Anschließend werden zwei Treatment-Variablen näher untersucht, die eher grundsätzliche Einstellungen der Befragten repräsentieren.

Arbeitslose, die von Befragten betreut werden, die die „grundsätzlich schnelle Wiedereingliederung“ präferierten, weisen zum Zeitpunkt von 90 Tagen nach Beginn ihrer Arbeitslosigkeit eine um 3,1 Prozentpunkte erhöhte Wahrscheinlichkeit auf, aus der Arbeitslosigkeit abzugehen (zweite Zeile in Abb. 2). Allerdings ist dieses Ergebnis nur auf dem 10\%-Niveau signifikant, und nach diesem Zeitraum sind keinerlei statistisch signifikanten Effekte festzustellen. Anders ist das zeitliche Muster beim Übergang in Beschäftigung. Hier steigt der Effekt kontinuierlich auf bis zu 4,6 Prozentpunkte zehn Monate nach Beginn der Arbeitslosigkeit an. Die Betreuung durch einen primär an einer schnellen Vermittlung orientierten Vermittler hat insofern auch für den Zeitraum noch Relevanz, in dem von einer schnellen Vermittlung

\footnotetext{
${ }^{26}$ Wird die Stichprobe in einer Robustheitsanalyse auf diejenigen Arbeitslosen begrenzt, die maximal 30 Tage nach Beginn der Arbeitslosigkeit dem befragten Vermittler zugeordnet waren, zeigen sich tendenziell schwächer ausgeprägte Ergebnisse.
}

Ergebnisvariable: Aufnahme einer svpfl. Beschäftigung

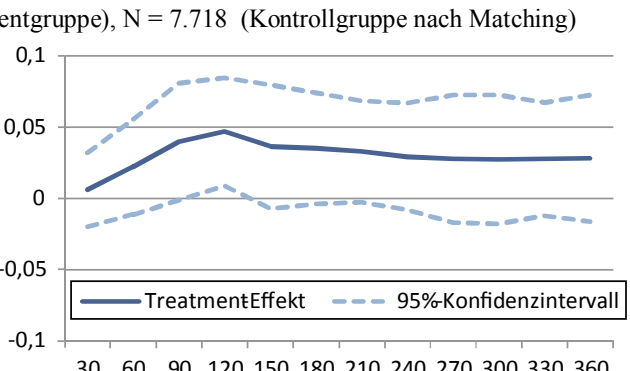

$30 \quad 60 \quad 90120150180210240270300330360$

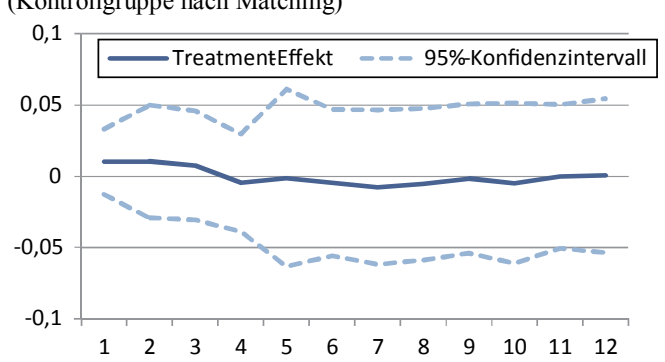

eigentlich nicht mehr die Rede sein kann. Der positive Effekt entspricht den Ergebnissen von Lagerström (2011), Kluve (2010) sowie Card et al. (2010), denen zufolge direkt auf Vermittlung abzielende Aktivitäten (anstatt einer Zuweisung in Maßnahmen) positiv auf die Beschäftigungschancen wirken. Allerdings ist der Treatment-Effekt nur schwach signifikant und erwies sich nicht in allen Robustheitsanalysen als stabil. ${ }^{27}$

Vermittler, die die Verantwortung für die Arbeitslosigkeit eher bei den Betroffenen selbst statt durch die gesellschaftlichen Verhältnisse verursacht sehen, erzielen zu Beginn der Arbeitslosigkeit ihrer Kunden höhere Abgangsraten aus diesem Zustand (dritte Zeile der Abb. 2). Der positive Treatment-Effekt erreicht zwei Monate nach Beginn der Arbeitslosigkeit eine Größenordnung von bis zu 4,7 Prozentpunkten. Kein klarer Effekt ist dagegen bei der Integration in sozialversicherungspflichtige Beschäftigung festzustellen. Offenbar wirkt die Einstellung zur Verursachung von Arbeitslosigkeit zwar auf den Zugang in Maßnahmen und andere Ereignisse, die Arbeitslosigkeit (temporär) beenden, führt aber nicht zu einer vermehrten Aufnahme einer sozialversicherungspflichtigen Beschäftigung.

Die nun folgenden vier Variablen enthalten Informationen zu detaillierteren Aspekten der Vermittlertätigkeit; sie beziehen sich auf die Einstellung der Befragten gegenüber Sperrzeiten, die Berücksichtigung von Wünschen der Betreuten bei der Vergabe von Maßnahmen, die Beurteilung des Stel-

\footnotetext{
${ }^{27}$ Dies gilt für die Einschränkung der Stichprobe auf Arbeitslosigkeitsepisoden, die mit einer zeitlichen Distanz von maximal einem halben Jahr zur Befragung der Vermittler begannen.
} 
lenwertes von Regelwerken sowie den Grad der subjektiven Autonomieneigung der Vermittler.

Bei der Einstellung der Befragten gegenüber Sperrzeiten zeigen sich gegenläufige Effekte bei den beiden Ergebnisvariablen (vierte Zeile der Abb. 2). Arbeitslose, deren Vermittler Sperrzeiten nicht nur im Notfall anwenden, gehen zwei Monate nach Beginn ihrer Arbeitslosigkeit mit höherer Wahrscheinlichkeit aus der Arbeitslosigkeit ab als Arbeitslose, deren Vermittler Sperrzeiten zurückhaltender vergeben. Im Gegensatz dazu verringert sich die Wahrscheinlichkeit einer Aufnahme von sozialversicherungspflichtiger Beschäftigung; der negative Effekt ist allerdings zu keinem Zeitpunkt signifikant von null verschieden. Dies steht im Gegensatz zur Studie von Hofmann (2012), die einen positiven Effekt von Sperrzeiten auf den Übergang in Beschäftigung findet; allerdings betrachtet sie die Wirkung einer individuellen Sanktionsvergabe. ${ }^{28}$ Der Unterschied zwischen den beiden Ergebnisvariablen könnte dadurch erklärt werden, dass ein verstärkter Rückgriff des Vermittlers auf Sperrzeiten die Arbeitslosen veranlasst, Maßnahmen anzutreten, um sich aus der Arbeitslosigkeit abzumelden. ${ }^{29}$ Zudem könnten Arbeitslose infolge der Androhung von Sperrzeiten auch vermehrt in die Nichterwerbstätigkeit abgehen.

Die Berücksichtigung von Wünschen der Arbeitslosen bei der Maßnahmenvergabe hat - im Gegensatz zu den Ergebnissen von Behncke et al. (2010a) - keine Wirkung auf die beiden Ergebnisvariablen im ersten Jahr der Arbeitslosigkeit (fünfte Zeile der Abb. 2). Tendenziell übereinstimmende Effekte auf das Ende der Arbeitslosigkeit und den Übergang in Beschäftigung sind für die Bedeutung von sonstigen internen Weisungen (jenseits der Handlungsprogramme) der BA festzustellen (sechste Zeile der Abb. 2). Arbeitslose, die von Vermittlern betreut werden, für die Weisungen bei der Maßnahmevergabe gegenüber anderen Kriterien wie der Verfügbarkeit der Maßnahme, individuelle Problemlagen der Arbeitslosen oder Zielvorgaben eine hohe Bedeutung haben, wechseln etwas häufiger in ein Beschäftigungsverhältnis als andere Arbeitslose. Die Effekte sind insgesamt aber gering; bei der Aufnahme einer Beschäftigung zeigt sich im vierten Monat nach Beginn der Arbeitslosigkeit ein signifikanter Effekt. Vor oder nach diesem Zeitpunkt sind keine signifikanten Wirkungen festzustellen, wobei die Effekte auf die Beschäftigung bis sieben Monate nach Beginn der Arbeitslosigkeit an der Schwelle zur statistischen Signifikanz bleiben. Damit besteht schwache Evidenz dafür, dass interne Weisungen der BA, die zunächst

\footnotetext{
${ }^{28}$ Boockmann et al. (2009) knüpfen an die Sanktionsneigung der Jobcenter an. Auch sie finden - allerdings für den SGB-II-Bereich - einen positiven Effekt von Sanktionen auf die Integration in Beschäftigung.

${ }^{29}$ Boockmann und Neumann (2012) finden, dass von Vermittlern, die Sanktionen nicht nur ausnahmsweise verhängen, insgesamt mehr Maßnahmen vergeben werden. Überraschenderweise ist der Effekt am größten bei der Förderung der Selbständigkeit und nicht bei kurzfristigen Maßnahmen wie z. B. Bewerbungstrainings. Hinsichtlich dieser Frage besteht weiterer Forschungsbedarf.
}

rasche und umfassende Integrationsbemühungen (Eigenbemühungen Kunde, Stellensuchlauf durch Vermittler) vor dem Einsatz weiterer Maßnahmen vorschreiben, kurzfristig die Integrationschancen verbessern. ${ }^{30}$ Allerdings gilt auch hier, dass die Treatment-Effekte in einigen Robustheitsanalysen schwächer ausgeprägt waren.

Beim subjektiven Autonomiebedarf der Vermittler - verortet zwischen den extremen Aussagen „Regeln und Handlungsanweisungen sind unabdingbar notwendige Grundlagen für meine Arbeit“ bis zu „Um meine tägliche Arbeit erfolgreich machen zu können, brauche ich völlige Entscheidungsfreiheit" - ergeben sich dagegen keine signifikanten Wirkungen (siebte Zeile der Abb. 2). Offenbar wirken bezüglich der Handlungsspielräume der Vermittler eher die objektiven Gegebenheiten als die subjektive Wahrnehmung. ${ }^{31}$

Obwohl jeweils nur eine Treatment-Variable verwendet wird und teilweise Korrelationen zwischen den TreatmentVariablen bestehen (siehe Tab. 4), ist nicht anzunehmen, dass die wenigen signifikanten Effekte einer Treatment-Variablen auf die Nichtberücksichtigung anderer Treatment-Variablen zurückzuführen ist. Denn die Effekte der mit den signifikanten Variablen korrelierenden Treatment-Variablen sind ihrerseits stets insignifikant. Beispielsweise kann die Wirkung der Bedeutung interner Weisungen auf die Aufnahme einer sozialversicherungspflichtigen Beschäftigung nicht durch den Effekt der ausgelassenen Variable „Entscheidungsfreiheit“ hervorgerufen werden, da der Treatment-Effekt der letztgenannten Variable in der Größenordnung nur sehr gering und statistisch nicht von null verschieden ist.

\section{Schlussfolgerungen}

In dieser Studie werden - erstmals für Deutschland - Befragungsdaten von Arbeitsvermittlern mit Daten über die von ihnen betreuten Arbeitslosen zusammengespielt, um zu überprüfen, ob die Arbeitsmarktchancen der Betreuten von Strategien und Einstellungen der Vermittler abhängen. Ein wichtiges Ergebnis für die künftige Durchführung ähnlicher Studien ist die technische Durchführbarkeit einer solchen Verknüpfung.

In der Befragung, die im Jahr 2009 in zehn Agenturen für Arbeit durchgeführt wurde, wurde eine Vielzahl von möglichen Vorgehensweisen erhoben. Wir verwendeten Informationen über Handlungsweisen und Einstellungen, die sich in

\footnotetext{
${ }^{30}$ Solche internen Weisungen dienen dazu, Ressourcen effizient einzusetzen, Geschäftsprozesse zu unterstützen und den Rahmen für eine einheitliche Rechtsanwendung zu gestalten. Beispielsweise regeln sonstige Weisungen den Einsatz von Fördermaßnahmen wie Maßnahmen bei Trägern, Arbeitgebern, der freien Förderung oder auch die Vergabe von Eingliederungszuschüssen.

${ }^{31}$ Werden bestimmte Vermittlereigenschaften im Propensity Score berücksichtigt, fällt der Effekt der Weisung schwächer und der Effekt des Autonomiebedarfs stärker aus.
} 
der Literatur als bedeutsam erwiesen haben, als TreatmentVariablen. Die Anwendung des statistischen MatchingVerfahrens ist in diesem Anwendungsfall besonders gut begründet, weil in der Befragung auch erhoben wurde, wie die Arbeitslosen den einzelnen Vermittlern zugeteilt wurden. Dies geschieht überwiegend auf der Basis beobachteter Faktoren, insbesondere des Berufs. Diese Zuteilung wird im Matching-Verfahren berücksichtigt und so lässt sich annehmen, dass die verbliebenen Unterschiede in der Verteilung der Arbeitslosen auf die Vermittler zufällig sind.

Alles in allem kann die empirische Analyse nur ausnahmsweise statistisch gesicherte Wirkungen aufgrund von Handlungsspielräumen der Vermittler aufdecken. In den Ergebnissen zeichnet sich jedoch eine Tendenz ab, wonach Vermittler, die häufigen Kontakt zu ihren Kunden haben, stärker auf eine schnelle Vermittlung setzen und die Kunden im Hinblick auf den Arbeitsmarkt und weniger hinsichtlich ihrer persönlichen Situation beraten, bessere Ergebnisse hinsichtlich der Beendigung von Arbeitslosigkeit und der Integration in Beschäftigung erzielen als andere. Dabei sind die Ergebnisse nicht einheitlich. Zudem bleibt die Größenordnung der Treatment-Effekte auf die Wahrscheinlichkeit eines Übergangs stets unterhalb von fünf Prozentpunkten. Ein zweites, jedoch ebenfalls in der Ausprägung eher begrenztes Ergebnis betrifft die Wirkung stärkerer Vorgaben. Wo sonstige interne Weisungen bei der Maßnahmenvergabe eine größere Rolle spielen, erhöhen sich die Übergangsraten leicht. Auch dieses Ergebnis ist allerdings höchstens an der Schwelle zur statistischen Signifikanz und trifft nur für die ersten Monate der Arbeitslosigkeit zu. Es gilt zudem nicht für andere Vorgaben wie die im
Befragungszeitraum geltenden Handlungsprogramme und Zielvorgaben. Auch hier bleiben die geschätzten TreatmentEffekte in der Größenordnung unterhalb von fünf Prozentpunkten. Beim Vergleich der Größenordnung dieser Effekte mit den Wirkungen arbeitsmarktpolitischer Maßnahmen muss berücksichtigt werden, dass im hier betrachteten Fall der Übernahme der erfolgreichen Strategien keine direkten Kosten gegenüberstehen. Insofern kann auch eine Wirkung von wenigen Prozentpunkten als nicht zu vernachlässigender Effekt angesehen werden. Wenn die erfolgreichen Strategien mit erhöhtem Einsatz von arbeitsmarktpolitischen Maßnahmen einhergehen, beispielsweise einer erhöhten Nutzung von Eingliederungszuschüssen, kann die Anwendung bestimmter Strategien allerdings indirekt Mehrkosten verursachen.

Insgesamt bestätigt sich durch die aufgefundene Varianz in den Befragungsdaten, dass trotz der vorhandenen Regelwerke Entscheidungsspielräume für die Durchführung der öffentlichen Arbeitsvermittlung verbleiben. Zudem zeigt sich, dass der damit entstehende individuelle Einfluss der Vermittler für den Erfolg der Arbeitsförderung teilweise mitbestimmend ist. Daher sollten bei der Konzeption künftiger Maßnahmen und Änderungen im Bereich des SGB III die Wirkungen auf die Handlungsspielräume der Vermittler noch stärker berücksichtigt werden.

Danksagung Wir bedanken uns bei Peter Bartelheimer, Tobias Brändle, Katrin Harsch, Peter Kupka, Gesine Stephan, Joachim Wilde sowie zwei anonymen Gutachtern und dem betreuenden Herausgeber, Reinhard Hujer, für hilfreiche Kommentare. Ferner danken wir Susanne Koch, Martin Rosemann, Hans Verbeek und den Projektmitarbeiterinnen und -mitarbeitern des IAB für wertvolle Vorarbeiten. 


\section{Appendix}

Tab. 5 Einbezogene Agenturen für Arbeit. (Quelle: Auswahl und Zuordnung auf Basis von Dauth et al. (2008))

\begin{tabular}{|c|c|}
\hline $\begin{array}{l}\text { Agentur } \\
\text { für Arbeit }\end{array}$ & IAB-Regionaltyp \\
\hline 1 & $\begin{array}{l}\text { Typ Ib: Bezirke in Ostdeutschland mit schlechten } \\
\text { Arbeitsmarktbedingungen }\end{array}$ \\
\hline 2 & $\begin{array}{l}\text { Typ Ic: Bezirke i.d. R in Ostdeutschland mit hoher } \\
\text { Arbeitslosigkeit, z. T. in Randlage zum Westen }\end{array}$ \\
\hline 3 & $\begin{array}{l}\text { Typ IIa: Großstädtisch geprägte Bezirke mit hoher } \\
\text { Arbeitslosigkeit }\end{array}$ \\
\hline 4 & $\begin{array}{l}\text { Typ IIb: Vorwiegend großstädtisch geprägte Bezirke mit } \\
\text { mäßig hoher Arbeitslosigkeit }\end{array}$ \\
\hline 5 & $\begin{array}{l}\text { Typ IIIa: Bezirke mit überdurchschnittlicher Arbeitslo- } \\
\text { sigkeit, aber mäßiger Dynamik }\end{array}$ \\
\hline 6 & $\begin{array}{l}\text { Typ IIIa: Bezirke mit überdurchschnittlicher Arbeitslo- } \\
\text { sigkeit, aber mäßiger Dynamik }\end{array}$ \\
\hline 7 & $\begin{array}{l}\text { Typ IV: Zentren mit günstiger Arbeitsmarktlage und } \\
\text { hoher Dynamik }\end{array}$ \\
\hline 8 & $\begin{array}{l}\text { Typ IV: Zentren mit günstiger Arbeitsmarktlage und } \\
\text { hoher Dynamik }\end{array}$ \\
\hline 9 & $\begin{array}{l}\text { Typ Vb: Mittelstandsstrukturierte Bezirke mit günstiger } \\
\text { Arbeitsmarktlage }\end{array}$ \\
\hline 10 & $\begin{array}{l}\text { Typ Vc: Bezirke mit günstigster Arbeitsmarktlage und } \\
\text { hoher Dynamik }\end{array}$ \\
\hline
\end{tabular}

Tab. 6 Mittelwerte und Standardabweichungen der Kontrollvariablen. (Quelle: IAB-Befragung von Vermittlern und Fallmanagern im Jahr 2009. Administrative Daten der BA. Eigene Berechnungen)

\begin{tabular}{|c|c|c|}
\hline Variable/Definition & Mittelwert & Std.-Abw. \\
\hline $\begin{array}{l}\text { BE25: Anteil Beschäftigtenzeiten in den } \\
\text { letzten zehn Jahren, erstes Quartil }\end{array}$ & 0,253 & 0,435 \\
\hline $\begin{array}{l}\text { BE75: Anteil Beschäftigtenzeiten in den } \\
\text { letzten zehn Jahren, drittes Quartil }\end{array}$ & 0,247 & 0,431 \\
\hline $\begin{array}{l}\text { ALO25: Anteil Arbeitslosigkeitszeiten in } \\
\text { den letzten zehn Jahren, erstes Quartil }\end{array}$ & 0,259 & 0,438 \\
\hline $\begin{array}{l}\text { ALO75: Anteil Arbeitslosigkeitszeiten in } \\
\text { den letzten zehn Jahren, drittes Quartil }\end{array}$ & 0,249 & 0,433 \\
\hline $\begin{array}{l}\text { NE25: Anteil Nichterwerbstätigkeitszeiten } \\
\text { in den letzten zehn Jahren, erstes Quartil }\end{array}$ & 0,254 & 0,436 \\
\hline $\begin{array}{l}\text { NE75: Anteil Nichterwerbstätigkeitszeiten } \\
\text { in den letzten zehn Jahren, drittes Quartil }\end{array}$ & 0,248 & 0,432 \\
\hline $\begin{array}{l}\text { PRG25: Anteil Arbeitsmarktprogramm- } \\
\text { zeiten in den letzten zehn Jahren, erstes } \\
\text { Quartil }\end{array}$ & 0,479 & 0,500 \\
\hline $\begin{array}{l}\text { PRG 75: Anteil Arbeitsmarktprogramm- } \\
\text { zeiten in den letzten zehn Jahren, drittes } \\
\text { Quartil }\end{array}$ & 0,255 & 0,436 \\
\hline Interaktion Altersdummy ab 35 mit BE25 & 0,345 & 0,475 \\
\hline Interaktion Altersdummy ab 35 mit BE75 & 0,248 & 0,432 \\
\hline Interaktion Altersdummy ab 35 mit ALO25 & 0,431 & 0,495 \\
\hline Interaktion Altersdummy ab 35 mit ALO75 & 0,252 & 0,434 \\
\hline Interaktion Altersdummy ab 35 mit NE25 & 0,775 & 0,418 \\
\hline Interaktion Altersdummy ab 35 mit NE75 & 0,225 & 0,418 \\
\hline Interaktion Altersdummy ab 35 mit ASU25 & 0,643 & 0,479 \\
\hline Interaktion Altersdummy ab 35 mit ASU75 & 0,251 & 0,434 \\
\hline Interaktion Altersdummy ab 35 mit PRG25 & 0,542 & 0,498 \\
\hline Interaktion Altersdummy ab 35 mit PRG75 & 0,247 & 0,431 \\
\hline
\end{tabular}

Tab. 6 (Fortsetzung)

Variable/Definition

$\begin{array}{ll}\text { Mittelwert } & \text { Std.-Abw. } \\ 0,171 & 0,377 \\ 0,144 & 0,351 \\ 0,162 & 0,369 \\ 0,153 & 0,360 \\ 0,209 & 0,407 \\ 0,310 & 0,463 \\ 0,310 & 0,463 \\ 0,295 & 0,456 \\ 0,748 & 0,434 \\ 0,208 & 0,406 \\ 0,034 & 0,180 \\ & \\ 0,089 & 0,285 \\ 0,088 & 0,284 \\ 0,049 & 0,215 \\ 0,468 & 0,499 \\ 0,100 & 0,300 \\ & \\ 0,564 & 0,496 \\ 0,014 & 0,116 \\ 0,020 & 0,141\end{array}$

Alter: 30-34

Alter: $35-39$

(n)

Alter: $40-44$

Alter: 45-49

Alter: ab 50

Hauptschulabschluss

Mittlere Reife

Abitur

Fachausbildung

Hochschule

Staatsangehörigkeit: EU und andere

Industrieländer

Staatsangehörigkeit: Rest der Welt

Nicht verheiratet, lebt in Partnerschaft

Alleinerziehend

Verheiratet

Gesundheitliche Einschränkungen als

Vermittlungshemmnis

Geschlecht

Schwerbehindert oder gleichgestellt

Berufe in der Land-, Tier- und

Forstwirtschaft

Metall-, Maschinenbau und verwandte

Berufe

Montierer, Metallberufe usw.

0,040

0,195

Hoch- und Tiefbauberufe

Ausbauberufe, Holz- und

0,174

Kunststoffverarbeitung

Techniker

Warenkaufleute

Dienstleistungskaufleute

Verkehrsberufe

Organisations-, Verwaltungs- und

Büroberufe

Ordnung-, Sicherheitsberufe, sonstige

Dienstleistungen

Künstlerische, schriftwerkschaffende und

Sozialberufe

Gesundheitsberufe

0,188

0,037

0,188

Agentur 1

0,037

$\begin{array}{ll}0,036 & 0,186 \\ 0,078 & 0,268\end{array}$

$0,043 \quad 0,204$

$0,108 \quad 0,310$

$0,173 \quad 0,378$

$0,096 \quad 0,294$

$0,106 \quad 0,308$

Agentur 2

Agentur 3

Agentur 4

Agentur 5

Agentur 6

Agentur 7

Agentur 8

Agentur 9
$0,067 \quad 0,250$

$0,075 \quad 0,263$

$0,081 \quad 0,273$

$0,197 \quad 0,398$

$0,126 \quad 0,331$

$0,163 \quad 0,370$

$0,030 \quad 0,170$

$0,074 \quad 0,262$

$0,039 \quad 0,193$

$0,029 \quad 0,167$ 
Tab. 7 Koeffizienten aus der Schätzung der Propensity Scores für die einzelnen Strategien. (Quelle: IAB-Befragung von Vermittlern und Fallmanagern im Jahr 2009. Administrative Daten der BA. Eigene Berechnungen)

\begin{tabular}{|c|c|c|c|c|c|c|c|}
\hline Variable/Definition & $\begin{array}{l}\text { Kontakt- } \\
\text { dichte }\end{array}$ & $\begin{array}{l}\text { Schnelle } \\
\text { Wiederein- } \\
\text { gliederung }\end{array}$ & $\begin{array}{l}\text { Individuelle } \\
\text { Verantwor- } \\
\text { tung } \\
\end{array}$ & $\begin{array}{l}\text { Sperrzeiten } \\
\text { nicht nur im } \\
\text { Notfall }\end{array}$ & $\begin{array}{l}\text { Eingehen auf } \\
\text { Wünsche des } \\
\text { Arbeitslosen }\end{array}$ & $\begin{array}{l}\text { Ermessens- } \\
\text { lenkende } \\
\text { Weisungen } \\
\end{array}$ & $\begin{array}{l}\text { Entschei- } \\
\text { dungsfrei- } \\
\text { heit }\end{array}$ \\
\hline \multirow{2}{*}{$\begin{array}{l}\text { BE25: Anteil Beschäftigtenzeiten in den } \\
\text { letzten zehn Jahren, erstes Quartil }\end{array}$} & 0,0375 & $-0,0398$ & $-0,0269$ & $-0,0337$ & $-0,0498$ & $-0,0353$ & 0,0583 \\
\hline & 1,35 & $-1,64$ & $-0,99$ & $-1,33$ & $-1,95$ & $-1,47$ & 1,74 \\
\hline \multirow{2}{*}{$\begin{array}{l}\text { BE75: Anteil Beschäftigtenzeiten in den } \\
\text { letzten zehn Jahren, drittes Quartil }\end{array}$} & $-0,0187$ & $-0,0723 *$ & $-0,0336$ & 0,0478 & $-0,0305$ & $-0,00589$ & $0,135^{* *}$ \\
\hline & $-0,51$ & $-2,23$ & $-0,93$ & 1,4 & $-0,88$ & $-0,18$ & 3,01 \\
\hline \multirow{2}{*}{$\begin{array}{l}\text { ALO25: Anteil Arbeitslosigkeitszeiten in den } \\
\text { letzten zehn Jahren, erstes Quartil }\end{array}$} & 0,0217 & $-0,018$ & $-0,0423$ & $-0,0873 * * *$ & $-0,0302$ & $-0,037$ & $-0,0682$ \\
\hline & 0,75 & $-0,71$ & $-1,5$ & $-3,38$ & $-1,15$ & $-1,49$ & $-1,94$ \\
\hline \multirow{2}{*}{$\begin{array}{l}\text { ALO75: Anteil Arbeitslosigkeitszeiten in den } \\
\text { letzten zehn Jahren, drittes Quartil }\end{array}$} & $-0,0402$ & $0,0965 * * *$ & 0,0249 & $-0,0144$ & 0,0142 & 0,0452 & $-0,0535$ \\
\hline & $-1,36$ & 3,78 & 0,87 & $-0,52$ & 0,52 & 1,74 & $-1,57$ \\
\hline \multirow{2}{*}{$\begin{array}{l}\text { NE25: Anteil Nichterwerbstätigkeitszeiten in } \\
\text { den letzten zehn Jahren, erstes Quartil }\end{array}$} & $-0,0464$ & 0,022 & $-0,0488$ & $-0,0358$ & 0,0114 & $0,127^{* *}$ & 0,0193 \\
\hline & $-1,04$ & 0,57 & $-1,11$ & $-0,86$ & 0,27 & 3,16 & 0,37 \\
\hline \multirow{2}{*}{$\begin{array}{l}\text { NE75: Anteil Nichterwerbstätigkeitszeiten in } \\
\text { den letzten zehn Jahren, drittes Quartil }\end{array}$} & 0,041 & 0,0138 & 0,0159 & 0,0371 & $-0,0207$ & $-0,0365$ & $-0,0284$ \\
\hline & 1,35 & 0,52 & 0,54 & 1,35 & $-0,75$ & $-1,4$ & $-0,79$ \\
\hline \multirow{2}{*}{$\begin{array}{l}\text { PRG25: Anteil Arbeitsmarktprogrammzeiten } \\
\text { in den letzten zehn Jahren, erstes Quartil }\end{array}$} & $0,0856^{*}$ & $-0,0593$ & $-0,0448$ & $-0,0695^{*}$ & $-0,0283$ & $-0,0972 * *$ & $-0,0364$ \\
\hline & 2,38 & $-1,89$ & $-1,3$ & $-2,12$ & $-0,85$ & $-3,14$ & $-0,86$ \\
\hline \multirow{2}{*}{$\begin{array}{l}\text { PRG 75: Anteil Arbeitsmarktprogrammzeiten } \\
\text { in den letzten zehn Jahren, drittes Quartil }\end{array}$} & 0,033 & 0,0333 & $-0,0297$ & $-0,0713 * *$ & $-0,0248$ & 0,0484 & $-0,0238$ \\
\hline & 1,1 & 1,29 & $-1,03$ & $-2,61$ & $-0,91$ & 1,85 & $-0,69$ \\
\hline \multirow[t]{2}{*}{ Interaktion Altersdummy ab 35 mit BE25 } & $-0,0215$ & 0,0347 & 0,0873 & 0,0441 & $-0,0652$ & $-0,0059$ & 0,0346 \\
\hline & $-0,27$ & 0,51 & 1,21 & 0,63 & $-0,93$ & $-0,09$ & 0,39 \\
\hline \multirow[t]{2}{*}{ Interaktion Altersdummy ab 35 mit BE75 } & 0,0143 & $-0,00199$ & 0,0563 & $-0,0469$ & $0,0857 *$ & 0,0192 & $-0,0788$ \\
\hline & 0,32 & $-0,05$ & 1,3 & $-1,15$ & 2,08 & 0,49 & $-1,48$ \\
\hline \multirow[t]{2}{*}{ Interaktion Altersdummy ab 35 mit ALO25 } & $-0,053$ & 0,0641 & 0,0182 & 0,00158 & 0,00766 & $-0,0406$ & $-0,0244$ \\
\hline & $-1,34$ & 1,88 & 0,47 & 0,05 & 0,22 & $-1,2$ & $-0,51$ \\
\hline \multirow[t]{2}{*}{ Interaktion Altersdummy ab 35 mit ALO75 } & 0,0522 & $-0,0162$ & $-0,0207$ & $-0,0465$ & 0,00824 & $-0,0114$ & 0,0167 \\
\hline & 1,47 & $-0,53$ & $-0,6$ & $-1,42$ & 0,25 & $-0,37$ & 0,41 \\
\hline \multirow[t]{2}{*}{ Interaktion Altersdummy ab 35 mit NE25 } & 0,00363 & $-0,031$ & 0,0446 & $-0,0152$ & $-0,041$ & $-0,0454$ & $-0,0226$ \\
\hline & 0,08 & $-0,74$ & 0,94 & $-0,34$ & $-0,91$ & $-1,04$ & $-0,39$ \\
\hline \multirow[t]{2}{*}{ Interaktion Altersdummy ab 35 mit NE75 } & $-0,0616$ & $-0,033$ & $0,0703 *$ & $0,0637 *$ & 0,0132 & $-0,0189$ & 0,0172 \\
\hline & $-1,9$ & $-1,18$ & 2,23 & 2,15 & 0,45 & $-0,67$ & 0,46 \\
\hline \multirow[t]{2}{*}{ Interaktion Altersdummy ab 35 mit ASU25 } & $-0,006$ & $0,0483^{*}$ & 0,00475 & 0,00989 & 0,0104 & 0,0141 & $-0,0459$ \\
\hline & $-0,24$ & 2,26 & 0,2 & 0,44 & 0,46 & 0,66 & $-1,59$ \\
\hline \multirow[t]{2}{*}{ Interaktion Altersdummy ab 35 mit PRG25 } & $-0,061$ & 0,0776 & 0,0161 & 0,0299 & $0,123 * *$ & 0,0738 & $-0,0538$ \\
\hline & $-1,28$ & 1,86 & 0,35 & 0,68 & 2,78 & 1,77 & $-0,96$ \\
\hline \multirow[t]{2}{*}{ Interaktion Altersdummy ab 35 mit PRG75 } & $-0,0584$ & $-0,0273$ & $-0,0247$ & 0,0124 & 0,0173 & $-0,0277$ & $-0,036$ \\
\hline & $-1,53$ & $-0,82$ & $-0,66$ & 0,36 & 0,5 & $-0,83$ & $-0,82$ \\
\hline \multirow[t]{2}{*}{ Alter: $30-34$} & $-0,0412$ & $-0,0398$ & $-0,0358$ & $0,0823 * *$ & 0,0318 & 0,00672 & $-0,0566$ \\
\hline & $-1,34$ & $-1,47$ & $-1,2$ & 2,92 & 1,11 & 0,25 & $-1,52$ \\
\hline \multirow[t]{2}{*}{ Alter: $35-39$} & $-0,0822$ & 0,102 & 0,081 & 0,103 & $-0,0194$ & $-0,0139$ & $-0,0451$ \\
\hline & $-0,77$ & 1,11 & 0,81 & 1,09 & $-0,2$ & $-0,15$ & $-0,36$ \\
\hline \multirow[t]{2}{*}{ Alter: $40-44$} & $-0,101$ & 0,126 & 0,0734 & 0,111 & 0,0139 & 0,000163 & $-0,0764$ \\
\hline & $-0,96$ & 1,38 & 0,74 & 1,18 & 0,15 & 0 & $-0,62$ \\
\hline Alter: $45-49$ & $-0,112$ & 0,165 & 0,133 & 0,0757 & 0,00548 & 0,00974 & $-0,0812$ \\
\hline & $-1,07$ & 1,81 & 1,34 & 0,8 & 0,06 & 0,11 & $-0,66$ \\
\hline Alter: ab 50 & $-0,1$ & 0,125 & 0,087 & 0,105 & $-0,0598$ & $-0,0173$ & $-0,0742$ \\
\hline & $-0,96$ & 1,37 & 0,88 & 1,12 & $-0,63$ & $-0,19$ & $-0,61$ \\
\hline Hauptschulabschluss & $-0,0292$ & $-0,0244$ & $-0,0556$ & $-0,0547$ & $-0,0174$ & $0,0816^{* *}$ & $-0,0302$ \\
\hline & $-0,85$ & $-0,8$ & $-1,65$ & $-1,65$ & $-0,52$ & 2,68 & $-0,76$ \\
\hline Mittlere Reife & 0,00689 & 0,042 & $-0,0785^{*}$ & $-0,0899 * *$ & $-0,0418$ & $0,154 * * *$ & $-0,0415$ \\
\hline & 0,19 & 1,3 & $-2,21$ & $-2,59$ & $-1,19$ & 4,75 & $-0,98$ \\
\hline Abitur & $-0,112 * *$ & $-0,00267$ & $-0,036$ & 0,00233 & $-0,0773^{*}$ & $0,157 * * *$ & $-0,240 * * *$ \\
\hline
\end{tabular}


Tab. 7 (Fortsetzung)

\begin{tabular}{|c|c|c|c|c|c|c|c|}
\hline Variable/Definition & $\begin{array}{l}\text { Kontakt- } \\
\text { dichte }\end{array}$ & $\begin{array}{l}\text { Schnelle } \\
\text { Wiederein- } \\
\text { gliederung }\end{array}$ & $\begin{array}{l}\text { Individuelle } \\
\text { Verantwor- } \\
\text { tung }\end{array}$ & $\begin{array}{l}\text { Sperrzeiten } \\
\text { nicht nur im } \\
\text { Notfall } \\
\end{array}$ & $\begin{array}{l}\text { Eingehen auf } \\
\text { Wünsche des } \\
\text { Arbeitslosen }\end{array}$ & $\begin{array}{l}\text { Ermessens- } \\
\text { lenkende } \\
\text { Weisungen }\end{array}$ & $\begin{array}{l}\text { Entschei- } \\
\text { dungsfrei- } \\
\text { heit }\end{array}$ \\
\hline & $-2,78$ & $-0,08$ & $-0,94$ & 0,06 & $-2,04$ & 4,45 & $-5,19$ \\
\hline \multirow[t]{2}{*}{ Fachausbildung } & $-0,0265$ & $-0,0178$ & $-0,0309$ & $-0,0787$ & 0,0144 & 0,0631 & $-0,013$ \\
\hline & $-0,54$ & $-0,42$ & $-0,66$ & $-1,68$ & 0,31 & 1,46 & $-0,24$ \\
\hline \multirow[t]{2}{*}{ Hochschule } & $-0,132 *$ & $-0,796^{* * *}$ & $-0,772 * * *$ & $-0,288 * * *$ & $0,671 * * *$ & $-0,346^{* * *}$ & $1,609 * * *$ \\
\hline & $-2,27$ & $-15,79$ & $-13,6$ & $-5,39$ & 12,58 & $-6,93$ & $-22,75$ \\
\hline \multirow{2}{*}{$\begin{array}{l}\text { Staatsangehörigkeit: EU und andere } \\
\text { Industrieländer }\end{array}$} & $-0,0238$ & $-0,0287$ & $0,159 * * *$ & $0,303 * * *$ & 0,0798 & $-0,0668$ & $0,182 * *$ \\
\hline & $-0,49$ & $-0,66$ & 3,41 & 6,95 & 1,81 & $-1,58$ & 3,21 \\
\hline \multirow[t]{2}{*}{ Staatsangehörigkeit: Rest der Welt } & 0,0445 & $-0,0318$ & $0,173 * * *$ & $0,211 * * *$ & $-0,125 * * *$ & $-0,0131$ & $0,189 * * *$ \\
\hline & 1,37 & $-1,09$ & 5,5 & 6,96 & $-4,02$ & $-0,46$ & 5,03 \\
\hline \multirow[t]{2}{*}{ Nicht verheiratet, lebt in Partnerschaft } & 0,0202 & $0,0747 * *$ & 0,00887 & $-0,0484$ & $-0,0215$ & 0,0118 & $0,120 * *$ \\
\hline & 0,62 & 2,65 & 0,28 & $-1,64$ & $-0,72$ & 0,42 & 3,12 \\
\hline \multirow[t]{2}{*}{ Alleinerziehend } & $-0,0246$ & $-0,0443$ & $-0,0215$ & $-0,0739$ & 0,00465 & 0,0144 & 0,0327 \\
\hline & $-0,54$ & $-1,17$ & $-0,53$ & $-1,88$ & 0,12 & 0,38 & 0,67 \\
\hline \multirow[t]{2}{*}{ Verheiratet } & $-0,00728$ & 0,0268 & $-0,0251$ & $-0,122 * * *$ & $0,0808 * * *$ & $0,0809 * * *$ & $-0,0192$ \\
\hline & $-0,36$ & 1,52 & $-1,28$ & $-6,63$ & 4,35 & 4,6 & $-0,8$ \\
\hline \multirow{2}{*}{$\begin{array}{l}\text { Gesundheitliche Einschränkungen als } \\
\text { Vermittlungshemmnis }\end{array}$} & 0,0276 & 0,00229 & $-0,0506$ & $-0,0868^{*}$ & 0,0408 & 0,0121 & $-0,108^{*}$ \\
\hline & 0,74 & 0,07 & $-1,36$ & $-2,43$ & 1,17 & 0,36 & $-2,43$ \\
\hline \multirow[t]{2}{*}{ Geschlecht } & $-0,0155$ & $-0,0687^{* * *}$ & $-0,121 * * *$ & $-0,0248$ & $0,0550 * *$ & $0,0600 * * *$ & $-0,0283$ \\
\hline & $-0,73$ & $-3,79$ & $-6,02$ & $-1,33$ & 2,9 & 3,33 & $-1,14$ \\
\hline \multirow[t]{2}{*}{ Schwerbehindert oder gleichgestellt } & $0,651 * * *$ & $0,301 * * *$ & $-0,385^{* * *}$ & $-0,220 * *$ & $0,487 * * *$ & $-0,533 * * *$ & $0,326^{* * *}$ \\
\hline & 8,24 & 4,51 & $-4,39$ & $-2,99$ & 6,78 & $-8,07$ & 3,65 \\
\hline \multirow{2}{*}{$\begin{array}{l}\text { Berufe in der Land-, Tier- und } \\
\text { Forstwirtschaft }\end{array}$} & $0,518^{* * *}$ & $0,173^{* *}$ & $-0,457 * * *$ & 0,0382 & $0,224 * * *$ & 0,086 & $-0,380 * * *$ \\
\hline & 8,19 & 3,19 & $-6,74$ & 0,62 & 3,71 & 1,55 & $-5,2$ \\
\hline \multirow{2}{*}{$\begin{array}{l}\text { Metall-, Maschinenbau und verwandte } \\
\text { Berufe }\end{array}$} & $-0,148 * *$ & $-0,565^{* * *}$ & $-0,478 * * *$ & $0,154^{* *}$ & $-0,308 * * *$ & $0,595 * * *$ & $-0,0151$ \\
\hline & $-2,95$ & $-12,61$ & $-8,98$ & 3,14 & $-6,46$ & 12,62 & $-0,24$ \\
\hline \multirow[t]{2}{*}{ Montierer, Metallberufe usw, } & $-0,504 * * *$ & $-0,527 * * *$ & $-0,195 * *$ & $-0,654 * * *$ & $0,493 * * *$ & $0,422 * * *$ & $-0,400 * * *$ \\
\hline & $-8,54$ & $-10,63$ & $-3,25$ & $-11,45$ & 9,51 & 8,08 & $-5,87$ \\
\hline \multirow[t]{2}{*}{ Hoch- und Tiefbauberufe } & $0,361 * * *$ & $0,201 * * *$ & $-0,455^{* * *}$ & 0,0776 & $-0,495 * * *$ & $0,384 * * *$ & $0,664 * * *$ \\
\hline & 7,16 & 4,46 & $-8,44$ & 1,49 & $-9,24$ & 8,24 & 10,73 \\
\hline \multirow{2}{*}{$\begin{array}{l}\text { Ausbauberufe, Holz- und } \\
\text { Kunststoffverarbeitung }\end{array}$} & $0,376^{* * *}$ & 0,0741 & $-0,380 * * *$ & $0,560 * * *$ & $-0,214 * * *$ & $0,169 * * *$ & $0,453 * * *$ \\
\hline & 7,82 & 1,65 & $-7,17$ & 10,63 & $-4,27$ & 3,73 & 7,73 \\
\hline \multirow[t]{2}{*}{ Techniker } & $0,284 * * *$ & $-0,301 * * *$ & $-0,188 * * *$ & $0,469 * * *$ & $-0,0397$ & $0,223 * * *$ & $-0,123$ \\
\hline & 5,44 & $-6,11$ & $-3,39$ & 9,05 & $-0,78$ & 4,82 & $-1,7$ \\
\hline \multirow[t]{2}{*}{ Warenkaufleute } & $-0,712 * * *$ & $0,762 * * *$ & $0,586 * * *$ & $0,305^{* * *}$ & $-0,333 * * *$ & $0,153 * * *$ & $-0,507 * * *$ \\
\hline & $-16,68$ & 21,13 & 16,09 & 8,28 & $-8,78$ & 4,37 & $-9,83$ \\
\hline \multirow[t]{2}{*}{ Dienstleistungskaufleute } & $-0,529 * * *$ & $-0,0706$ & 0,0448 & $0,129 * *$ & $-0,833^{* * *}$ & $0,323 * * *$ & $-0,650 * * *$ \\
\hline & $-9,74$ & $-1,65$ & 0,98 & 2,9 & $-16,83$ & 7,21 & $-10,06$ \\
\hline \multirow[t]{2}{*}{ Verkehrsberufe } & $-0,00709$ & $-0,123 * * *$ & $-0,396 * * *$ & $-0,205 * * *$ & $0,202 * * *$ & $0,204 * * *$ & $0,109 * *$ \\
\hline & $-0,21$ & $-3,94$ & $-11,01$ & $-6,11$ & 6,08 & 6,52 & 2,6 \\
\hline \multirow[t]{2}{*}{ Organisations-, Verwaltungs- und Büroberufe } & $-0,183 * * *$ & 0,00211 & $-0,199 * * *$ & $-0,0487$ & $-0,359 * * *$ & $0,356 * * *$ & $-0,710 * * *$ \\
\hline & $-5,1$ & 0,07 & $-5,78$ & $-1,54$ & $-11,04$ & 11,52 & $-16,54$ \\
\hline \multirow{2}{*}{$\begin{array}{l}\text { Ordnung-, Sicherheitsberufe, sonstige } \\
\text { Dienstleistungen }\end{array}$} & $0,209 * * *$ & $0,308 * * *$ & $0,0785^{*}$ & $-0,158 * * *$ & $0,109 * *$ & $-0,143 * * *$ & $-0,430 * * *$ \\
\hline & 5,69 & 9,51 & 2,25 & $-4,59$ & 3,22 & $-4,46$ & $-10,66$ \\
\hline
\end{tabular}


Tab. 7 (Fortsetzung)

\begin{tabular}{|c|c|c|c|c|c|c|c|}
\hline Variable/Definition & $\begin{array}{l}\text { Kontakt- } \\
\text { dichte }\end{array}$ & $\begin{array}{l}\text { Schnelle } \\
\text { Wiederein- } \\
\text { gliederung }\end{array}$ & $\begin{array}{l}\text { Individuelle } \\
\text { Verantwor- } \\
\text { tung }\end{array}$ & $\begin{array}{l}\text { Sperrzeiten } \\
\text { nicht nur im } \\
\text { Notfall }\end{array}$ & $\begin{array}{l}\text { Eingehen auf } \\
\text { Wünsche des } \\
\text { Arbeitslosen }\end{array}$ & $\begin{array}{l}\text { Ermessens- } \\
\text { lenkende } \\
\text { Weisungen }\end{array}$ & $\begin{array}{l}\text { Entschei- } \\
\text { dungsfrei- } \\
\text { heit }\end{array}$ \\
\hline \multirow{2}{*}{$\begin{array}{l}\text { Künstlerische, schriftwerkschaffende und } \\
\text { Sozialberufe }\end{array}$} & $0,248 * * *$ & $-0,00141$ & $-0,129 * * *$ & $-0,157 * * *$ & $-0,515^{* * *}$ & $0,243 * * *$ & $-0,00446$ \\
\hline & 6,14 & $-0,04$ & $-3,29$ & $-4,36$ & $-13,98$ & 7,11 & $-0,1$ \\
\hline \multirow[t]{2}{*}{ Gesundheitsberufe } & 0,0459 & $0,392 * * *$ & $0,168 * * *$ & $-0,375^{* * *}$ & $-0,163 * * *$ & 0,0742 & $0,166^{* * *}$ \\
\hline & 1 & 10,05 & 4,12 & $-9,11$ & $-4,05$ & 1,92 & 3,36 \\
\hline \multirow[t]{2}{*}{ Dummy für Ausbildung: fehlende Angabe } & $-0,00923$ & $-0,00639$ & $-0,00222$ & $-0,053$ & 0,035 & $-0,0714$ & $-0,00144$ \\
\hline & $-0,19$ & $-0,15$ & $-0,05$ & $-1,1$ & 0,74 & $-1,62$ & $-0,03$ \\
\hline \multirow{2}{*}{$\begin{array}{l}\text { Dummy für Staatsanghörigkeit: fehlende } \\
\text { Angabe }\end{array}$} & 0,275 & $0,617 *$ & 0,386 & 0,0226 & 0,427 & $-0,0424$ & $-0,0825$ \\
\hline & 1,04 & 2,32 & 1,43 & 0,09 & 1,66 & $-0,17$ & $-0,24$ \\
\hline \multirow{2}{*}{$\begin{array}{l}\text { Dummy für gesundheitliche Einschränkun- } \\
\text { gen: fehlende Angabe }\end{array}$} & 0,0108 & 0,0364 & 0,0326 & $-0,0168$ & 0,0344 & 0,0248 & $-0,0702 *$ \\
\hline & $-0,43$ & 1,67 & 1,35 & $(-0,75)$ & 1,52 & 1,16 & $(-2,39)$ \\
\hline \multirow{2}{*}{$\begin{array}{l}\text { Dummy für Schwerbehinderung: fehlende } \\
\text { Angabe }\end{array}$} & & $-1,007$ & & 0,0601 & & 0,6 & $-0,711$ \\
\hline & & $-1,46$ & & 0,1 & & 0,87 & $-0,93$ \\
\hline \multirow[t]{2}{*}{ Agentur 1} & $0,798 * * *$ & $-0,0721 *$ & $-0,677 * * *$ & $0,228 * * *$ & $-0,198 * * *$ & $0,614 * * *$ & $-0,396 * * *$ \\
\hline & 19,22 & $-2,06$ & $-15,16$ & 7,02 & $-5,92$ & 17,68 & $-9,94$ \\
\hline \multirow[t]{2}{*}{ Agentur 2} & $1,979 * * *$ & $0,191 * * *$ & $0,252 * * *$ & $0,526^{* * *}$ & $-0,632 * * *$ & $-0,03$ & \\
\hline & 50,97 & 5,56 & 7,09 & 16,09 & $-18,98$ & $-0,93$ & \\
\hline \multirow[t]{2}{*}{ Agentur 3} & $0,756^{* * *}$ & $0,794 * * *$ & $-0,0948 * * *$ & $0,202 * * *$ & $-1,135^{* * *}$ & $0,427 * * *$ & $-1,490 * * *$ \\
\hline & 23,11 & 30,55 & $-3,36$ & 8,04 & $-41,28$ & 16,77 & $-42,39$ \\
\hline \multirow[t]{2}{*}{ Agentur 4} & $1,699 * * *$ & $0,811 * * *$ & $-0,0286$ & $1,372 * * *$ & $-1,229 * * *$ & $0,272 * * *$ & \\
\hline & 49,7 & 28,17 & $-0,9$ & 42,76 & $-37,8$ & 9,61 & \\
\hline \multirow[t]{2}{*}{ Agentur 5} & $0,572 * * *$ & $-0,0791 * *$ & $-0,0610^{*}$ & $-0,155^{* * *}$ & $-0,229 * * *$ & 0,0101 & $-0,678 * * *$ \\
\hline & 17,06 & -3 & $-2,18$ & $-5,99$ & $-8,96$ & 0,4 & $-23,35$ \\
\hline \multirow[t]{2}{*}{ Agentur 6} & & $0,948 * * *$ & & & & $-0,314 * * *$ & $0,913 * * *$ \\
\hline & & 19,5 & & & & $-7,16$ & 16,32 \\
\hline \multirow[t]{2}{*}{ Agentur 7} & $2,505^{* * *}$ & $-0,107 * *$ & $-0,189 * * *$ & & & $0,752 * * *$ & \\
\hline & 60,21 & $-3,09$ & -5 & & & 20,78 & \\
\hline \multirow[t]{2}{*}{ Agentur 8} & & & & & $0,311 * * *$ & & $-0,588 * * *$ \\
\hline & & & & & 7,36 & & $-13,45$ \\
\hline \multirow[t]{2}{*}{ Agentur 9} & & $-0,0725$ & $0,621 * * *$ & $0,0997 *$ & $0,137 * *$ & & \\
\hline & & $-1,48$ & 13,25 & 2,16 & 2,98 & & \\
\hline
\end{tabular}

Die Tabelle enthält die Ergebnisse von sechs Probit-Modells mit den sechs einzelnen Strategien als abhängigen Variablen. In den Zeilen sind die Koeffizienten abgetragen, t-Statistiken stehen jeweils darunter. *, ** und *** bezeichnen signifikante Koeffizienten zum 5-\%-, 1-\%- und 0,1-\%-Niveau. Referenzkategorien für die Dummy-Variablen: Zurückliegende Zeiten von Beschäftigung, Arbeitslosigkeit, Nichterwerbstätigkeit oder Maßnahmen: jeweils 2. Quartil; Alter: Altersgruppe 25-30; schulische Ausbildung: ohne Hauptschulabschluss; berufliche Ausbildung: ohne beruflichen Abschluss oder Hochschulabschluss; Staatsangehörigkeit: deutsch; Familienstand: alleinlebend; Geschlecht: weiblich; Gesundheitliche Einschränkungen: keine; Schwerbehindert oder gleichgestellt: nein; Beruf: sonstige/ohne nähere Angabe

\section{Literatur}

Abadie, A., Imbens, G.W.: Large sample properties of matching estimators for average treatment effects. Econometrica 74(1), 235267 (2006)

Arni, P., Lalive, R., van Ours, J.C.: "How effective are unemployment benefit sanctions? Looking beyond unemployment exit." Journal of Applied Econometrics 28(7), 1153-1178 (2013)

Behncke, S., Frölich; M., Lechner, M.: Unemployed and their caseworkers: should they be friends or foes? J. of the Royal Statistical Society Series A 173(1), 67-92 (2010a)
Behncke, S., Frölich, M., Lechner, M.: A caseworker like me - does the similarity between the unemployed and their caseworkers increase job placements? Econ. J. 120(549), 1430-1459 (2010b)

van den Berg, G.J., van der Klaauw, B.: Job search monitoring and sanctions - a brief survey of some recent results. IFAU Report 2005:8 (2005)

van den Berg, G.J., van der Klaauw, B., van Ours, J.C.: Punitive sanctions and the transition rate from welfare to work. J. Labor Econ. 22(1), 211-210 (2004)

van den Berg, G.J., Kjærsgaard, L., Rosholm, M.: To meet or not to meet (Your case worker) - that is the question. IZA Discussion Paper No. 6476 (2012) 
Blien, U., Hirschenauer, F.: Vergleichstypen 2005. Neufassung der Regionaltypisierung für Vergleiche zwischen Agenturbezirken. IABForschungsbericht 24 (2005)

Boockmann, B., Neumann, M.: Attitudes and autonomy: caseworker influence on access to ALMP programs in Germany. Manuskript, IAW Tübingen (2012)

Boockmann, B., Thomsen, S.L., Walter, T.: Intensifying the use of benefit sanctions: an effective tool to shorten welfare receipt and speed up transitions to employment? IZA Discussion Paper No. 4580 (2009)

Boockmann, B., Koch, S., Rosemann, M., Stops, M., Verbeek, H.: Aktive Arbeitsmarktpolitik: Fördern und Fordern aus Sicht der Vermittler. IAB-Kurzbericht 25 (2010)

Boockmann, B., Osiander, C., Stops, M., Verbeek, H.: Effekte von Vermittlerhandeln und Vermittlerstrategien im SGB II und SGB III (Pilotstudie). Abschlussbericht an das Institut für Arbeitsmarktund Berufsforschung (IAB) durch das Institut für Angewandte Wirtschaftsforschung e. V. (IAW). IAB-Forschungsbericht 07 (2013). Online verfügbar unter: http://doku.iab.de/forschungsbericht/2013/fb0713.pdf

Brown, A.J.G., Koettl, J.: Active labor market programs: employment gain or fiscal drain? IZA Discussion Paper No. 6880 (2012)

Caliendo, M., Kopeinig, S.: Some practical guidance for the implementation of propensity score matching. In: J. Econ. Surv. 22(1), 31-72 (2008)

Card, D., Kluve, J., Weber, A.: Active labour market policy evaluations: a meta-analysis. Econ. J. 120(548), F452-F477 (2010)

Dauth, W., Hirschenauer, F., Rüb, F.: Vergleichstypen 2008: Neufassung der SGB-III-Typisierung. IAB-Forschungsbericht 08 (2008)

Eichhorst, W., Kaufmann, O., Konle-Seidl, R.: Bringing the jobless into work? Experiences with activation schemes in Europe and the US. Springer, Berlin/Heidelberg (2008)

Frölich, M., Lechner, M., Behncke, S., Hammer, S., Schmidt, N., Menegale, S., Lehmann, A., Iten, R.: Einfluss der RAV auf die Wiedereingliederung von Stellensuchenden. Studie im Auftrag der Aufsichtskommission für den Ausgleichsfonds der Arbeitslosenversicherung. SECO Publikation Arbeitsmarktpolitik 20 (1.2007). Staatssekretariat für Wirtschaft (SECO), Bern (2007)

Gautier P.A., Muller, P., van der Klauw, B., Rosholm, M., Svarer, M.: Estimating equilibrium effects of job search assistance. CEPR Discussion Paper No. 9066 (2012)

Hainmueller, J., Hofmann, B., Krug, G., Wolf, K.: Do lower caseloads improve the effectiveness of active labor market policies? New evidence from German employment offices. LASER Discussion Paper 52 (2011)

Heckman, J.J., LaLonde, R.J., Smith, J.A.: The economics and econometrics of active labor market programs. In: Ashenfelter, O., Card, D. (Hrsg.) Handbook of labor economics, volume III Part A, pp. 1865-2097. Elsevier, San Diego (1999)

Hofmann, B.: Short- and long-term ex-post effects of unemployment insurance sanctions - evidence from West Germany. Jahrbücher für Nationalökonomie und Statistik 232(1) 31-60 (2012)

Kastoryano, S., van der Klaauw, B.: Dynamic evaluation of job search assistance. IZA Discussion Paper No. 5424 (2011)

Kluve, J.: The effectiveness of European active labour market programs. Labour Econ. 17(6), 904-918 (2010)

Knuth, M., Schweer, O., Siemes, S.: Drei Menüs - und kein Rezept? Dienstleistungen am Arbeitsmarkt in Großbritannien, in den Niederlanden und in Dänemark. Diskussionspapier des Gesprächskreises Arbeit und Soziales der Friedrich-Ebert-Stiftung (2004)

de Koning, J.: The reform of the Dutch public employment service. SEOR Paper for the TLM meeting on active labour market policies, Rotterdam (2004)

Lagerström, J.: How important are caseworkers - and why? New evidence from Swedish employment offices. Institute for labour market policy evaluation (IFAU) Working Paper 2011:10 (2011)
Lechner, M., Wunsch, C.: Sensitivity of matching-based program evaluations to the availability of control variables. Labour Economics 21(C), 111-121 (2013)

Lipsky, M.: Street-level bureaucracy: dilemmas of the individual in public services. Sage, New York (1980)

Martin, J.P., Grubb, D.: What works and for whom: a review of OECD countries' experience with labour market policies. Swed. Econ. Policy Rev. 8(2), 9-56 (2001)

Müller, K.-U., Oschmiansky, F.: Die Sanktionspolitik der Arbeitsagenturen. Eine empirische Analyse zu Sperrzeiten. In: Schütz, H., Mosley, H. (Hrsg.) Arbeitsagenturen auf dem Prüfstand. Leistungsvergleich und Reformpraxis der Arbeitsvermittlung. Edition sigma, 95-135 (2005)

Oberschachtsiek, D., Scioch, P., Seysen, C., Heining, J.: Stichprobe der Integrierten Erwerbsbiografien IEBS - Handbuch für die IEBS in der Fassung 2008. FDZ Datenreport 03 (2009)

Pedersen, J.M., Rosholm, M., Svarer, M.: Experimental evidence on the effects of early meetings and activation. Working Paper (2012)

Schütz, H.: Reform der Arbeitsvermittlung. Uniformierungsdruck in der Bundesagentur für Arbeit. Budrich, Opladen (2008)

Sell, S.: Modernisierung und Professionalisierung der Arbeitsvermittlung. Strategien, Konzepte und Modelle unter Berücksichtigung internationaler Erfahrungen. Gutachten der Friedrich-Ebert-Stiftung, Bonn (2006)

Svarer, M.: The effect of sanctions on the exit rate from unemployment. Evidence from Denmark. Economica 78(312), 751-778 (2011)

Vaut, S.: Umbau der BA. In: Jann, W., Schmid, G. (Hrsg.) Eins zu Eins? Eine Zwischenbilanz der Hartzreformen am Arbeitsmarkt. Edition sigma, Berlin, 63-75 (2004)

Weatherall, C.D., Markwardt, K.S.: Caseworker Behavior and Clients' Employability. Danish National Centre for Social Research - Research Department of Social Policy and Welfare Services. Working Paper 04:2010 (2010)

Weishaupt, J.T.: A silent revolution? New management ideas and the reinvention of European public employment services. SocioEcon. Rev. 8(3), 461-486 (2010)

Wunsch, C.: Optimal use of labor market policies: the role of job search assistance. Review of Economics and Statistics 9(3), 1030-1045 (2013)

Prof. Dr. Bernhard Boockmann ist wissenschaftlicher Direktor am Institut für Angewandte Wirtschaftsforschung (IAW) in Tübingen. In seinen wissenschaftlichen Arbeiten befasst er sich mit Arbeitsmarktfragen und dabei insbesondere mit der Evaluation von Arbeitsmarkpolitik, individuellen Erwerbsverläufen, der Wirkung von Arbeitsmarktregulierungen und der Lohnbildung.

Christopher Osiander studierte Sozialwissenschaften an der Friedrich-Alexander-Universität Erlangen-Nürnberg. Seit 2009 ist er wissenschaftlicher Mitarbeiter am Institut für Arbeitsmarkt- und Berufsforschung in Nürnberg. Seine Forschungsinteressen sind Weiterbildungsentscheidungen und Evaluation von Qualifizierungsmaßnahmen sowie das Vermittlungshandeln der Bundesagentur für Arbeit.

Michael Stops ist Diplom-Volkswirt und Diplom-Verwaltungswirt (FH). Derzeit arbeitet er als wissenschaftlicher Mitarbeiter bei der Forschungsgruppe Berufliche Arbeitsmärkte am Institut für Arbeitsmarktund Berufsforschung (IAB) in Nürnberg. In seiner Forschung befasst er sich mit arbeitsmarktökonomischen Fragestellungen, insbesondere zu Marktausgleichsprozessen und die Wirkung institutioneller Veränderungen auf regionalen und beruflichen Teilarbeitsmärkten. 